\title{
From Prestellar to Protostellar Cores II. Time Dependence and Deuterium Fractionation
}

\author{
Y. Aikawa \\ Department of Earth and Planetary Sciences, Kobe University, 657-8501, Kobe, Japan \\ aikawa@kobe-u.ac.jp \\ V. Wakelam, F. Hersant \\ Univ. Bordeaux, LAB, UMR 5804, F-33270, Floirac, France \\ CNRS, LAB, UMR 5804, F-33270, Floirac, France \\ R.T. Garrod \\ Department of Astronomy, Cornell University, Ithaca, NY 14853, USA \\ and \\ E. Herbst \\ Departments of Chemistry, Astronomy, and Physics, University of Virginia, \\ Charlottesville, VA 22904, USA
}

\begin{abstract}
We investigate the molecular evolution and $\mathrm{D} / \mathrm{H}$ abundance ratios that develop as star formation proceeds from a dense-cloud core to a protostellar core, by solving a gas-grain reaction network applied to a 1-D radiative hydrodynamic model with infalling fluid parcels. Spatial distributions of gas and ice-mantle species are calculated at the first-core stage, and at times after the birth of a protostar. Gas-phase methanol and methane are more abundant than CO at radii $r \lesssim 100 \mathrm{AU}$ in the first-core stage, but gradually decrease with time, while abundances of larger organic species increase. The warm-up phase, when complex organic molecules are efficiently formed, is longer-lived for those fluid parcels in-falling at later stages. The formation of unsaturated carbon chains (warm carbon-chain chemistry) is also more effective in later stages; $\mathrm{C}^{+}$, which reacts with $\mathrm{CH}_{4}$ to form carbon chains, increases in abundance as the envelope density decreases. The large organic molecules and carbon chains are strongly deuterated, mainly due to high $\mathrm{D} / \mathrm{H}$ ratios in the parent molecules, determined
\end{abstract}


in the cold phase. We also extend our model to simulate simply the chemistry in circumstellar disks, by suspending the 1-D infall of a fluid parcel at constant disk radii. The species $\mathrm{CH}_{3} \mathrm{OCH}_{3}$ and $\mathrm{HCOOCH}_{3}$ increase in abundance in $10^{4}-10^{5}$ yr at the fixed warm temperature; both also have high $\mathrm{D} / \mathrm{H}$ ratios.

Subject headings: stars: formation, ISM: clouds, ISM: abundances

\section{Introduction}

In star-forming cores, temperature and density vary both temporally and spatially. The rates of chemical processes, which determine the molecular abundances in these cores, also change according to the physical conditions. In prestellar cores with high densities and low temperatures, various atoms and molecules are depleted onto grains, where they are hydrogenated by grain-surface reactions. In the later, protostellar phase, on the other hand, molecules are desorbed from the grains back into the gas phase in the central hightemperature regions. The molecular abundances in the cores thus change dramatically in the course of star formation.

It is well established that molecular clouds are generally not in chemical equilibrium; the chemical timescale is comparable or longer than the dynamical timescale of the clouds. For example, the adsorption timescale of gaseous species onto grain surfaces in cold prestellar cores is

$$
\left[\pi a^{2} \sqrt{\frac{8 k T}{\pi m}} S n_{\text {grain }}\right]^{-1} \sim 1 \times 10^{6}\left(\frac{10^{4} \mathrm{~cm}^{-3}}{n_{\mathrm{H}}}\right)\left(\frac{10^{-12}}{n_{\text {grain }} / n_{\mathrm{H}}}\right)\left(\frac{10^{-5} \mathrm{~cm}}{a}\right)^{2}\left(\frac{1.0}{S}\right)[\mathrm{yr}],
$$

where $a$ and $n_{\text {grain }}$ are the radius and number density of the grain particles, respectively, and $S$ is the sticking probability (e.g. Herbst 1993). At the typical density of molecular clouds, $n_{\mathrm{H}} \sim 10^{4} \mathrm{~cm}^{-3}$, this timescale is comparable to the free-fall timescale of a cold core (e.g. Spitzer 1978)

$$
t_{\mathrm{ff}}=\sqrt{\frac{3 \pi}{32 G \rho}} \sim 4.3 \times 10^{5}\left(\frac{10^{4} \mathrm{~cm}^{-3}}{n_{\mathrm{H}}}\right)^{1 / 2}[\mathrm{yr}] .
$$

Thus, hydrodynamic-chemical models are required to investigate theoretically the abundances and spatial distributions of molecules in star-forming cores.

Since the hydro-chemical models combine hydrodynamics and chemical reaction-network models, there is a variety of previous work related to this topic. The chemistry in prestellar cores has been investigated using Bonner-Ebert spheres and/or the isothermal collapse model (e.g. Aikawa et al. 2001; Keto \& Caselli 2010). The chemistry in protostellar cores 
is often investigated using a pseudo-time-dependent gas-phase chemistry model with a constant temperature $(\sim 200 \mathrm{~K})$ and density $\left(n_{\mathrm{H}} \sim 10^{6} \mathrm{~cm}^{-3}\right)$, in which a high abundance of ice sublimates is given as an initial condition (e.g. Charnley et al. 1992). While such simple models give chemical timescales of various species, which is useful in the interpretation of protostellar cores, ice abundances should ideally be determined by the gas-grain chemical model in the prestellar phase.

Rodgers \& Charnley (2003) investigated the chemistry in a protostellar core using the inside-out collapse model (Shu 1977); they calculated radial distributions of molecular abundances at $10^{2}, 10^{3}, 10^{4}$ and $10^{5} \mathrm{yr}$ after the collapse of the singular isothermal sphere. Temporal variation of the temperature distribution, which is a critical parameter for the chemistry, was adopted from Adams \& Shu (1985). The model shows the onion-skin type structure, in which more volatile species are sublimated to the gas phase at outer radii, with the sublimation radius moving outwards as the core becomes hotter. The model did not include a prestellar phase, since the inside-out collapse model corresponds to the main accretion phase, and the ice abundance was set as an initial condition.

Lee et al. (2004) went one step farther; they solved the chemistry in a core starting from a prestellar core and evolving to a protostellar core self-consistently. Their physical model of the core is a combination of a series of Bonner-Ebert spheres and inside-out collapse. The spatial and temporal variation of the temperature in the protostar is calculated by radiation transfer, assuming the luminosity of the central star as a function of time. The model clearly shows that the sublimation of ice (e.g. CO) affects the gas-phase chemistry significantly. Their chemical model, however, does not include grain-surface reactions except for $\mathrm{H}_{2}$ formation; dust-grain ice mantles are composed of molecules accreted directly from the gas phase.

Garrod \& Herbst (2006), on the other hand, constructed a comprehensive gas-grain chemical network with various grain-surface reactions. Their chemical network is solved with simple physical models in which the density is constant and the temperature increases with time. They showed that some large organic species are efficiently formed on grain surfaces starting at temperatures of 30-40 K and sublimated at higher temperatures. Their model accounts for the high abundance of large organic molecules such as methyl formate $\left(\mathrm{HCOOCH}_{3}\right)$ and dimethyl ether $\left(\mathrm{CH}_{3} \mathrm{OCH}_{3}\right)$ in low-mass protostellar cores, known as hot corinos (Ceccarelli et al. 2007). Aikawa et al. (2008), hereinafter Paper I, then applied the chemical network of Garrod \& Herbst (2006) to a 1-D radiation-hydrodynamic model of a star-forming core by Masunaga \& Inutsuka (2000). As expected, organic species such as $\mathrm{CH}_{3} \mathrm{CN}$ and $\mathrm{HCOOH}$ are formed in the warm-up phase and become abundant in the central region $(T \gtrsim 100 \mathrm{~K}$ ) of the protostellar core. In addition, the model shows that (unsaturated) 
carbon-chain species increase in abundance inwards at $T \sim 25 \mathrm{~K}$, which is observed as the so-called "Warm Carbon Chain Chemistry (WCCC)" (Sakai et al. 2008).

In this work, we update and improve the model of Paper I. The aim of the work is to investigate

1. the spatial distribution of molecules at several evolutionary stages from a prestellar core to a protostellar core,

2. the evolution of molecular $\mathrm{D} / \mathrm{H}$ ratios,

3. how the chemical composition in a disk differs from that in cores.

Paper I showed spatial distributions of molecules only at $9.3 \times 10^{4} \mathrm{yr}$ after the protostellar birth, although the model starts from a prestellar core. Here we show molecular distributions at assorted evolutionary stages to see how the distributions and peak abundances of hot corino species and carbon chains vary with time. We also extend the chemical reaction network to include both singly- and multiply-deuterated species. It is well established that molecular D/H ratios are enhanced by exothermic exchange reactions at low temperature. Once the star is formed, the core becomes warmer and the backward reactions (endothermicexchange reactions) become efficient. How the $\mathrm{D} / \mathrm{H}$ ratios vary after protostellar birth is of importance to link the observed high $\mathrm{D} / \mathrm{H}$ ratios in the ISM to the isotopic ratios observed in planetary matter such as comets and meteorites. In order to investigate such a link, it is also of great interest to determine how the chemical composition in a disk differs from that of cores. For the material in an infalling envelope, the duration of the warm-up phase and warm chemistry is determined by the size of the warm region divided by the free-fall velocity, which is rather short, as shown in Paper I. In circumstellar disks, on the other hand, material can stay in warm regions for longer timescales, since the disk is supported by rotation, at least partially. Because our model is spherical and thus does not include the structure and dynamics of disk formation, we briefly investigate the effect of prolonged warm temperature chemistry in the disk by calculating pseudo-time-dependent models with the initial abundances set by the infalling core model.

In the following section, we briefly describe our physical model of the star-forming core and the chemical reaction network $(\S 2)$. In $\S 3$, we report the spatial distribution of molecular abundances and $\mathrm{D} / \mathrm{H}$ ratios at assorted evolutionary stages. In $\S 4$, we discuss the effect on the chemistry of $2 \mathrm{D}$ structure in the core; we calculate a pseudo-time-dependent model of a warm disk-like phase. We also compare our model results with observational studies, and discuss the effect of grain-surface abstraction and substitution reactions on $\mathrm{D} / \mathrm{H}$ ratio of methanol. We summarize our conclusions in $\S 5$. 


\section{Model}

\subsection{Physical model of a star-forming core}

As in Paper I, we adopt the 1D (spherical) radiation hydrodynamic model of low-mass star formation by Masunaga, Mivama \& Inutsuka (1998) and Masunaga \& Inutsuka (2000). Initially, the central density of the molecular cloud core is $\rho=1.4 \times 10^{-19} \mathrm{~g} \mathrm{~cm}^{-3}$, which corresponds to a number density of hydrogen nuclei $n_{\mathrm{H}} \sim 6 \times 10^{4} \mathrm{~cm}^{-3}$. The outer boundary is fixed at $r=4 \times 10^{4} \mathrm{AU}$, so that the total mass is $3.852 M_{\odot}$, which exceeds the critical mass for gravitational instability. The contraction is almost isothermal as long as the cooling rate overwhelms the compressional heating, but eventually the latter dominates and the temperature rises in the central region. Afterwards, the first core, which is a hydrostatic core of $\mathrm{H}_{2}$ gas, is formed. When the central temperature reaches $\sim 2000 \mathrm{~K}$ (several hundred years after the first core formation), $\mathrm{H}_{2}$ starts to dissociate and the first core collapses. The central region becomes hydrostatic again when the dissociation and ionization of hydrogen are completed; this signifies the birth of a protostar. In our model, the prestellar core evolves to the protostellar core in $2.5 \times 10^{5} \mathrm{yr}$. After the birth of the protostar, the model further follows the evolution for $9.3 \times 10^{4} \mathrm{yr}$, during which the protostar grows by mass accretion from the envelope. At each evolutionary stage, the model gives the total luminosity of the core and the radial distribution of density, temperature, and infall velocity at $r \gtrsim 10^{-4}$ AU self-consistently. The original model by Masunaga \& Inutsuka (2000) included wavelength-dependent radiation transfer involving the opacities of the gas (Iglesias \& Rogers 1996; Alexander \& Ferguson 1994) and dust (Preibisch et al. 1993) to obtain both the temperatures of the gas and the various components of dust (silicate, carbon and ice) separately. For simplicity, we adopt their gas temperature and assume that it is equal to the dust temperature, since the differences between the gas and dust temperatures are not siginificant in the region we are interested in. Figure $1(a-c)$ shows the distribution of density, temperature, and infall velocity at assorted evolutionary stages, in which we present the molecular distribution in $\S 3: t=-5.6 \times 10^{2} \mathrm{yr}, 4.3 \times 10^{2} \mathrm{yr}$ and $9.3 \times 10^{4} \mathrm{yr}$. Here we define $t=0$ as the moment of the birth of a protostar. The total luminosity of the core is about $5.9 \times 10^{-3} L_{\odot}$ at $t=-5.6 \times 10^{2} \mathrm{yr}, 0.88 L_{\odot}$ at $t=4.3 \times 10^{2} \mathrm{yr}$, and $24 L_{\odot}$ at $9.3 \times 10^{4} \mathrm{yr}$. The first core is formed at around $t=-5.6 \times 10^{2} \mathrm{yr}$; Figure $1(a-c)$ shows that at this time a hydrostatic core of a few AU size is formed. It should be noted that the density in the envelope decreases with time after the protostar is formed, while the density increases with time in the prestellar stage. More detailed explanations of core evolution can

be found in Masunaga, Miyama \& Inutsuka (1998), Masunaga \& Inutsuka (2000) and Paper I.

Figure 1 $(d-e)$ shows the temporal variation of density and temperature in fluid parcels 
that fall to $r=2.5 \mathrm{AU}$ at $t=-5.6 \times 10^{2} \mathrm{yr}, 4.3 \times 10^{2} \mathrm{yr}$ and $9.3 \times 10^{4} \mathrm{yr}$. Naturally, the density and temperature increase with time. As can be seen in Eq. (2), the free-fall timescale is proportional to $\rho^{-1 / 2}$. While the core temperature rises in the central region, the infall velocity increases toward the center until the fluid parcel hits the surface of the central hydrostatic core (the first core or the second core). Hence the temporal variation of density and temperature accelerates in the infalling fluid parcels. In order to highlight the rapid rise near and at the final stage, the horizontal axis in Figure 1 $(d-e)$ is set to be the logarithm of $t_{2.5 \mathrm{AU}}-t$, where $t_{2.5 \mathrm{AU}}$ corresponds to $t=-5.6 \times 10^{2} \mathrm{yr}$ for the dotted line, $4.3 \times 10^{2} \mathrm{yr}$ for the dashed line, and $9.3 \times 10^{4} \mathrm{yr}$ for the solid line.

The temporal variation of temperature is critically important for chemistry. Garrod \& Herbst (2006) show that the grain-surface reactions of heavy-element radical species are efficient in a temperature range of $\approx 30-100 \mathrm{~K}$. Once the temperature gets higher than $\sim 100 \mathrm{~K}$, most volatile species are sublimated to the gas phase, where they begin to undergo gas-phase reactions. In Figure $10(d-e)$, we can easily read the timescale of these warm phases. For example, the fluid parcel that reaches $r=2.5 \mathrm{AU}$ at $t=9.3 \times 10^{4}$ yr stays at a temperature of $30-100 \mathrm{~K}$ for $\sim 10^{4} \mathrm{yr}$. After the fluid parcel gets warmer than $100 \mathrm{~K}$, it falls to the central star in a few $100 \mathrm{yr}$. The fluid parcels that reach $r=2.5 \mathrm{AU}$ at earlier time spend even less time in the warm regions.

In the original model by Masunaga \& Inutsuka (2000), the core starts contraction immediately, since the core mass exceeds the critical mass for gravitational instability. For our chemistry model, however, we need to set up the initial molecular abundances of this dense molecular core by assuming a simple history of fluid parcels before collapse. Since the formation of molecular cloud cores is out of the scope of this work, we simply assume that the core keeps its hydrostatic structure for $1 \times 10^{6} \mathrm{yr}$, implicitly assuming that turbulence supports it. In addition, we assume that the temperature in the pre-collapse phase is 10 $\mathrm{K}$, as typically observed in molecular clouds. After $1 \times 10^{6} \mathrm{yr}$, we lower the temperature smoothly but rapidly to the initial value in Masunaga \& Inutsuka (2000), $\sim 7 \mathrm{~K}$.

\subsection{Chemical reaction network}

We solve the rate equations of the gas-grain reaction network of Garrod \& Herbst (2006) under the time-dependent physical conditions of each fluid parcel, to obtain the radial distributions of molecular abundances both in the gas phase and ice mantles at assorted evolution-

ary stages. We use the gas-grain code Nautilus (Hersant et al. 2009; Semenov et al. 2010) to solve the rate equations. A layered structure of the ice mantle is not considered (see §3.2). The chemical reaction network model and parameters are essentially the same as in paper 
I, except for the following three updates: (i) Some gas-phase reaction rates are updated based on the KIDA database (http://kida.obs.u-bordeaux1.fr/). (ii) We included reactions between carbon-chain species with $\mathrm{H}$ atoms or $\mathrm{H}_{2}$ molecules, based on Harada et al. (2010). Hassel et al. (2011) showed that these reactions significantly change the evolution of radical carbon-chain species at temperatures $\gtrsim 100 \mathrm{~K}$. We do not, however, discriminate between isomers such as c- $\mathrm{C}_{3} \mathrm{H}_{2}$ and $\mathrm{H}_{2} \mathrm{CCC}$, which was done by Hassel et al. (2011). (iii) We include multi-deuterated species according to the following procedures.

First, the species list of the network model is extended to include mono-, doubly-, and triply-deuterated species. Then the reaction network, both in the gas phase and on grain surfaces, is extended to include these deuterated species. For example, if we have the reaction $\mathrm{AH}^{+}+\mathrm{BH} \rightarrow \mathrm{A}+\mathrm{BH}_{2}^{+}$in the original reaction list, we add

$\mathrm{AD}^{+}+\mathrm{BH} \rightarrow \mathrm{A}+\mathrm{BHD}^{+}$

$\mathrm{AH}^{+}+\mathrm{BD} \rightarrow \mathrm{A}+\mathrm{BHD}^{+}$

$\mathrm{AD}^{+}+\mathrm{BD} \rightarrow \mathrm{A}+\mathrm{BD}_{2}^{+}$

assuming that the rate coefficients are the same as for the original reaction. If there is more than one set of possible products,

$\mathrm{AHD}+\mathrm{BD} \rightarrow \mathrm{ABH}+\mathrm{D}_{2}$

$\mathrm{AHD}+\mathrm{BD} \rightarrow \mathrm{ABD}+\mathrm{HD}$,

for example, we set the branching ratio statistically (e.g. Furuya et al. 2011). In the above example, the branching ratio for the former and latter product channels is 1:2. We also assumed statistical branching ratios in dissociative recombination reactions, except for the recombination of deuterated $\mathrm{H}_{3}^{+}$; this assumption can result in underestimates of the molecular D/H ratio by a factor of two or so, since channels with a hydrogen atom as a product are more likely than analogous ones with a $\mathrm{D}$ atom due to the greater speed of the $\mathrm{H}$ atom (Le Petit \& Roueff 2003). For the recombination of deuterated $\mathrm{H}_{3}^{+}$, we adopted branching ratios listed in Roberts, Herbst \& Millar (2004). Indeed, these branching ratios are higher for reactions producing an $\mathrm{H}$ atom than the statistical value. For simplicity, we do not follow the position of the deuterium atoms in the reactions which include deuterated isomers. For example, in the reaction of $\mathrm{H}_{2} \mathrm{CO}+\mathrm{OD}$, the branching ratio of the three product channel

$\mathrm{H}_{2} \mathrm{CO}+\mathrm{OD} \rightarrow \mathrm{HCOOH}+\mathrm{D}$

$\mathrm{H}_{2} \mathrm{CO}+\mathrm{OD} \rightarrow \mathrm{DCOOH}+\mathrm{H}$

$\mathrm{H}_{2} \mathrm{CO}+\mathrm{OD} \rightarrow \mathrm{HCOOD}+\mathrm{H}$

is 1:1:1 in our current model, although the last branch would be the most probable in reality. So we do not aim to predict the abundance ratios of deuterated isomers.

Finally we included reactions listed in Millar, Bennet \& Herbst (1989) and Roberts, Herbst \& Millar (2004); these are mostly exchange reactions that cause the initial isotopic fractionation. At low temperatures, of several tens of Kelvin or less, deuterium fractionation is mainly trig- 
gered by $\mathrm{H}_{3}^{+}+\mathrm{HD} \rightarrow \mathrm{H}_{2} \mathrm{D}^{+}+\mathrm{H}_{2}$ (and its multi-deuterated analogues), which is exothermic by $230 \mathrm{~K}$. The effective rate of the backward reaction depends on the ortho/para ratio of $\mathrm{H}_{2}$, since the internal energy of ortho $\mathrm{H}_{2}$ helps overcome the endothermicity. But the ortho/para ratio of $\mathrm{H}_{2}$ is a complicated issue (e.g. Flower et al. 2006), and there is so far no observational evidence for a high abundance of ortho $\mathrm{H}_{2}$ in molecular clouds. Recently, Watanabe et al. (2010) measured the spin temperature of $\mathrm{H}_{2}$ formed on ice in the laboratory, obtaining a value greater than approximately $200 \mathrm{~K}$. But they also found that ortho $\mathrm{H}_{2}$ that is trapped on the ice surface is converted to para $\mathrm{H}_{2}$ after formation. Such conversion of ortho to para has never been considered in the prediction of the $\mathrm{o} / \mathrm{p}$ ratio of $\mathrm{H}_{2}$. In this work, we do not consider the o/p ratio; i.e. we assume the backward reaction of $\mathrm{H}_{3}^{+}+\mathrm{HD}$ is endothermic by $230 \mathrm{~K}$.

Inclusion of multi-deuterated species naturally increases the number of species and reactions. In total, our model includes 1564 species and 45371 reactions, while the original model includes 655 species and 6309 reactions.

As elemental abundances, we adopt the so-called "low-metal" values (Table 1 of Aikawa et al. (2001)). The species are assumed to be initially in the form of atoms or atomic ions except for hydrogen, which is entirely in its molecular form. The elemental $\mathrm{D} / \mathrm{H}$ ratio is set to $1.5 \times 10^{-5}$ (Piskunov et al. 1997). All deuterium resides initially in the HD molecule. The cosmic-ray ionization rate for $\mathrm{H}_{2}$ is set to $1.3 \times 10^{-17} \mathrm{~s}^{-1}$.

The sticking probability for gaseous neutral species onto grains is set to 0.5 , independent of temperature. Although the probability may be lower at high temperatures $(T \gtrsim$ $100 \mathrm{~K}$ ), the value of the sticking probability becomes unimportant once evaporation becomes dominant. We assume the same set of adsorption energies $\left(E_{\text {ads }}\right)$ as Garrod \& Herbst (2006), corresponding to a grain surface covered by water ice. The adsorption energies for deuterated species are set to the values used for their normal isotopes, with the exception of $\mathrm{D}$ atoms, whose adsorption energy is set $21 \mathrm{~K}$ higher $(471 \mathrm{~K})$ than that of $\mathrm{H}$ atoms $(450 \mathrm{~K})$, following Caselli et al. (2002). The grain-surface species can desorb to the gas phase via thermal evaporation and two non-thermal processes: cosmic-ray desorption (Hasegawa et al. 1993) and desorption via exothermic association reactions on grain surfaces (Williams 1968; Garrod \& Herbst 2006; Garrod et al. 2007). Recent laboratory work and molecular dynamics simulations indicate that photodesorption could also be important (Andersson \& van Dishoeck 2008; Öberg et al. 2009a, b; Munoz Caro et al. 2010; Arasa et al. 2011). There are several possible mechanisms for photodesorption: Molecules are dissociated into radicals and atoms, which then desorb separately to the gas phase; the dissociation products may first recombine on the grain and then desorb (Andersson \& van Dishoeck 2008); or the dissociation products may kick out neighboring ice-mantle species. Our model 
includes the second of these mechanisms, through the explicit inclusion of two separate processes; the photodissociation into radicals of grain-surface species by interstellar and cosmic ray-induced UV photons (see below), and the partial evaporation of the products of exothermic surface reactions (Garrod et al. 2007).

We assume the Langmuir-Hinshelwood mechanism for grain surface reactions; species can diffuse on grain surfaces by thermal hopping and react with each other when they meet. No quantum tunneling was assumed in the migration, even for $\mathrm{H}$ and $\mathrm{D}$ atoms (Garrod et al. 2007). The barrier for migration is set at $50 \%$ of the adsorption energy. We adopt the modified-rate approach for grain-surface reactions that include $\mathrm{H}$ and D atoms (Stantcheva et al. 2001; Caselli et al. 2002). In paper I, we did not adopt the modified-rate approach, since its significance is reduced in the model when assuming only thermal hopping of $\mathrm{H}$ atoms on the grain surface, compared with a model that includes quantum tunneling (Ruffle \& Herbst 2000). In the present work, however, the grain-surface reactions with D atoms could still be accretion-limited rather than migration-limited, and thus the modified rates can be important.

Extinction of interstellar UV radiation is calculated from the column density of hydrogen nuclei $\left(N_{\mathrm{H}}\right)$ from the core outer edge to each shell by the formulation $A_{\mathrm{V}}=N_{\mathrm{H}} /(1.59 \times$ $\left.10^{21} \mathrm{~cm}^{2}\right)$ mag. Initially, the visual extinction from the outer edge $\left(r=4 \times 10^{4} \mathrm{AU}\right)$ to the core center is about $5.5 \mathrm{mag}$, and the outermost shells in which we calculate chemistry are located at $1 \times 10^{4} \mathrm{AU}$, where $A_{\mathrm{V}} \sim 1 \mathrm{mag}$. Assuming that our model core is embedded in ambient clouds, we add $3 \mathrm{mag}$ to the visual extinction obtained above, and ignore photodissociation of $\mathrm{CO}$ and $\mathrm{H}_{2}$, which should be self- and mutually-shielded from the interstellar UV at $A_{\mathrm{v}} \gtrsim 1$ mag (e.g. van Dishoeck \& Black 1988). We also neglect the photodissociation of HD and $\mathrm{D}_{2}$, since it is not clear if and how much they are shielded by $\mathrm{H}_{2}$. This assumption would not much affect our results for molecular $\mathrm{D} / \mathrm{H}$ ratios, since the interstellar UV is extinguished by $\geq 3$ mag. The rates of photodissociation and photoionization by cosmic ray-induced UV radiation, on the other hand, are roughly independent of $A_{\mathrm{v}}$ and proportional to the cosmic-ray ionization rate, although the coefficients vary among molecular species. The photodissociation rates of $\mathrm{HD}$ and $\mathrm{D}_{2}$ by cosmic-ray induced $\mathrm{UV}$ are set to be the same as for $\mathrm{H}_{2}$. UV radiation from the central protostar is not considered in our model; since the model is $1 \mathrm{D}, \mathrm{UV}$ radiation is absorbed in close vicinity to the source. 


\section{Results}

\subsection{Temporal Variation of Molecular Distributions}

Figure $2(a-b)$ shows the radial distribution of simple molecules at $t=-5.6 \times 10^{2} \mathrm{yr}$, $4.3 \times 10^{2} \mathrm{yr}$, and $9.3 \times 10^{4} \mathrm{yr}$. These simple molecules comprise four major neutrals: $\mathrm{CO}$, $\mathrm{NH}_{3}, \mathrm{H}_{2} \mathrm{O}$ and $\mathrm{N}_{2}$. Major ions and electron abundance (i.e., the fractional ionization) are also plotted. A simple view of the radial distribution of major species is as follows: as the radius gets smaller, and the density higher, the species first tend to be depleted from the gas onto the ice. Finally, as the temperature rises, sublimation occurs and the species return rapidly and sharply to the gas at the so-called sublimation radius. Little change is seen in gas-phase values at yet smaller radii, due to the rapid collapse. Such a radial distribution of gas-phase abundance is consistent with the "drop abundance profile" derived from multi-line observations of $\mathrm{CO}$ and $\mathrm{H}_{2} \mathrm{O}$ toward protostars (e.g. Jørgensen 2004; Coutens et al. 2012). The sublimation radii of $\mathrm{CO}, \mathrm{NH}_{3}, \mathrm{H}_{2} \mathrm{O}$ and $\mathrm{N}_{2}$ increase with time, as the core temperature rises, so that the gas-phase material extends farther from the core. The $\mathrm{CO}$ depletion factor is defined as the ratio of the canonical value of $\mathrm{CO}$, which is close to the gaseous elemental abundance of carbon $\left(8 \times 10^{-5}\right.$ in our model $)$, to the actual abundance of gaseous CO. The depletion factor increases inward as the density increases, until it suddenly decreases at the sublimation radius. The peak value of the $\mathrm{CO}$ depletion factor decreases with time, because the density decreases. We note that the CO depletion factor does not reach unity even inside the sublimation radius in our model; i.e. CO abundance inside the sublimation radius is smaller than the canonical value, especially at early stages, because $\mathrm{CH}_{3} \mathrm{OH}$ and $\mathrm{CH}_{4}$ are slightly more abundant than CO. Low CO abundances inside the sublimation radius have recently been observed toward several protostars (Alonso-Albi et al. 2010; Yildiz et al. 2012; Fuente et al. 2012).

The fractional ionization decreases toward the center, because it is generally proportional

to $n_{\mathrm{H}}^{-1 / 2}$. It is noteworthy that $\mathrm{HCO}^{+}$, which is often used as a radio tracer of dense gas, decreases as two species with a higher proton affinity than $\mathrm{CO}\left(\mathrm{H}_{2} \mathrm{CO}\right.$ and $\left.\mathrm{NH}_{3}\right)$ sublime into the gas phase.

The distribution of complex organic species is shown in Figure 2 (c). At $t=-5.6 \times 10^{2}$ $\mathrm{yr}$, the temperature is lower than $\sim 10 \mathrm{~K}$ at $\gtrsim 100 \mathrm{AU}$ (Figure 1). In these cold regions, $\mathrm{CH}_{3} \mathrm{OH}$ ice is as abundant as $1.62 \times 10^{-5}$, and $\mathrm{HCOOH}$ ice and $\mathrm{CH}_{3} \mathrm{CN}$ ice are $\sim 10^{-9}$ relative to hydrogen nuclei. $\mathrm{CH}_{3} \mathrm{OH}$ ice is formed by the hydrogenation of $\mathrm{CO}$ ice, while $\mathrm{HCOOH}$ is mainly formed in the gas phase via the dissociative recombination of $\mathrm{CH}_{3} \mathrm{O}_{2}^{+}$, which is a product of the radiative-association reaction $\mathrm{HCO}^{+}+\mathrm{H}_{2} \mathrm{O}$. A fraction of the gas-phase $\mathrm{HCOOH}$ is adsorbed onto grains before subsequent gas-phase reactions. $\mathrm{CH}_{3} \mathrm{CN}$ 
is formed by association reactions (which add $\mathrm{C}$ and $\mathrm{H}$ to $\mathrm{CN}$ ) on grain surfaces. Because these species are formed by low-temperature chemistry, the abundances at this stage depend on the duration of the pre-collapse model assumed. $\mathrm{HCOOCH}_{3}$ ice, on the other hand, increases inwards at $10 \mathrm{AU}$; it is formed at warm temperatures, starting at 30-40 K.

At $t=4.3 \times 10^{2} \mathrm{yr}$, the warm region of $T \gtrsim 100 \mathrm{~K}$ expands to $\sim 10 \mathrm{AU}$, where complex organic species sublime. At $t=9.3 \times 10^{4} \mathrm{yr}$, many organic species are formed via grain surface reactions and gas phase reactions of sublimated species at $T \gtrsim 30 \mathrm{~K}$ (Garrod \& Herbst (2006); Paper I). Our model shows that the abundance of large organic species increases with time at the central region not only because the dust temperature exceeds their sublimation temperatures, but also because the duration of the warm chemistry is longer for fluid parcels in later stages than those in earlier stages (see Figure 1). It should also be noted that the abundance rise is not homogeneous; relative abundances among complex organic species (e.g. $\mathrm{CH}_{3} \mathrm{CHO} / \mathrm{HCOOCH}_{3}$ ) vary with time.

Figure 2 $2(d)$ shows the distribution of carbon-chain species. At $t=9.3 \times 10^{4} \mathrm{yr}$, carbon chains increase inwards at the sublimation radius of $\mathrm{CH}_{4}(T \sim 25 \mathrm{~K}, r \sim 2000 \mathrm{AU})$, showing that the chemistry is indeed described by WCCC; the $\mathrm{CH}_{4}$ reacts with $\mathrm{C}^{+}$to form $\mathrm{C}_{2} \mathrm{H}_{3}^{+}$, and unsaturated hydrocarbons are formed via subsequent gas-phase reactions and a partial contribution of grain surface hydrogenation (Sakai et al. (2008); Paper I; Hassel et al. (2008)). At earlier stages $\left(t=-5.6 \times 10^{2} \mathrm{yr}\right.$ and $\left.4.3 \times 10^{2} \mathrm{yr}\right)$, the formation of carbon-chains at the $\mathrm{CH}_{4}$ sublimation radius is much less significant. Although the $\mathrm{CH}_{4}$ abundance is as high as $2.5 \times 10^{-5}$, the other reactant to start $\mathrm{WCCC}, \mathrm{C}^{+}$, is deficient. Specifically, while the abundance of $\mathrm{C}^{+}$at the sublimation radius of $\mathrm{CH}_{4}$ is as high as $\sim 1 \times 10^{-10}$ at $t=9.3 \times 10^{4}$ $\mathrm{yr}$, it is $4 \times 10^{-15}$ at $t=-5.6 \times 10^{2} \mathrm{yr}$, because of the high density. Although some carbon chains are formed at the $\mathrm{CH}_{4}$ sublimation zone at $t=4.3 \times 10^{2} \mathrm{yr}$, the increment is much smaller than that at $t=9.3 \times 10^{4} \mathrm{yr}$. The abundances of carbon chains (e.g. $\left.\mathrm{C}_{3} \mathrm{H}_{2}\right)$ at the central region of these early phases are thus determined mostly by the sublimation of icy carbon chains formed in the cold era.

It should also be noted that $\mathrm{C}_{4} \mathrm{H}$ and $\mathrm{C}_{2} \mathrm{H}$ are depleted at radii less than a few hundred $\mathrm{AU}$ at $t=9.3 \times 10^{4} \mathrm{yr}$, which is consistent with observations of L1527 (Sakai et al. 2010). In these hot-temperature regions they react with $\mathrm{H}_{2}$ and are converted to $\mathrm{C}_{4} \mathrm{H}_{2}$ and $\mathrm{C}_{2} \mathrm{H}_{2}$, respectively (Hassel et al. 2011). $\mathrm{C}_{3} \mathrm{H}_{2}$, on the other hand, does not deplete at the central region, while Sakai et al. (2010) found that the cyclic form, $\mathrm{c}-\mathrm{C}_{3} \mathrm{H}_{2}$, does indeed exhibit a central dip. Hassel et al. (2011), who distinguish $\mathrm{c}^{-} \mathrm{C}_{3} \mathrm{H}_{2}$ from $\mathrm{H}_{2} \mathrm{CCC}$, also show that c$\mathrm{C}_{3} \mathrm{H}_{2}$ survives in hot regions $(\sim 100-200 \mathrm{~K})$ for several $10^{5} \mathrm{yr}$, while the carbene form is destroyed via reaction with $\mathrm{H}_{2}$ over a shorter time scale at $T \gtrsim 140 \mathrm{~K}$. Additional destruction paths for $\mathrm{c}^{-} \mathrm{C}_{3} \mathrm{H}_{2}$ are therefore required to account for the observations. 


\subsection{Molecular $\mathrm{D} / \mathrm{H}$ ratios}

Figure 3 shows the spatial distribution of selected mono-deuterated species, as well as $\mathrm{D}_{3}^{+}$, and their normal isotope counterparts at $t=-5.6 \times 10^{2} \mathrm{yr}, 4.3 \times 10^{2} \mathrm{yr}$ and $9.3 \times 10^{4} \mathrm{yr}$. The $\mathrm{D} / \mathrm{H}$ ratios of assorted molecules are shown in the bottom panels in Figure 3 . In lowtemperature chemistry $(T \sim 10 \mathrm{~K})$, gas-phase molecules are enriched with deuterium due to exothermic exchange reactions such as $\mathrm{H}_{3}^{+}+\mathrm{HD} \rightarrow \mathrm{H}_{2} \mathrm{D}^{+}+\mathrm{H}_{2}$ (and analogous reactions for multiply deuterated species; Millar, Bennett \& Herbst 1989; Roberts, Herbst \& Millar 2004). The backward reactions, e.g. $\mathrm{H}_{2} \mathrm{D}^{+}+\mathrm{H}_{2} \rightarrow \mathrm{H}_{3}^{+}+\mathrm{HD}$, are endothermic (typically by several $100 \mathrm{~K}$ ) and thus are negligible at low temperature $(\sim 10 \mathrm{~K})$. The $\mathrm{D} / \mathrm{H}$ ratio for

$\mathrm{H}_{3}^{+}$is not governed by these two reactions alone, as $\mathrm{H}_{3}^{+}$and its deuterated isotopomers are destroyed by reactions with $\mathrm{CO}$ and electrons. In the zone where gaseous $\mathrm{CO}$ is depleted, however, the $\mathrm{D} / \mathrm{H}$ ratio for $\mathrm{H}_{3}^{+}$is further enhanced. For example, at $t=-5.6 \times 10^{2} \mathrm{yr}$, $\mathrm{D}_{3}^{+}$becomes the major cation in the $\mathrm{CO}$ depletion zone at radii of a few tens to several hundred AU (see Fig. 3). Although the exothermic exchange reactions are limited to several species, the high $\mathrm{D} / \mathrm{H}$ ratio propagates to other molecules via gas-phase reactions; e.g. $\mathrm{H}_{2} \mathrm{D}^{+}$ gives its deuteron to other molecules and atoms via ion-molecule reactions. It is essential to include multi-deuterated species, such as $\mathrm{D}_{3}^{+}$, to calculate the molecular $\mathrm{D} / \mathrm{H}$ ratio, since the multiply-deuterated species can propagate D to other species more efficiently than can mono-deuterated ones. The high $\mathrm{D} / \mathrm{H}$ ratio also propagates to the grain surface; deuterated $\mathrm{H}_{3}^{+}$dissociatively recombines with electrons to produce $\mathrm{D}$ atoms, which are adsorbed onto grains and deuterate the ice-mantle species.

Once the core gets warmer than $\sim 20 \mathrm{~K}$, CO sublimation terminates the extreme deuteration $(\mathrm{XD} / \mathrm{XH} \gtrsim 1)$ of $\mathrm{H}_{3}^{+}$. At the same time, the endothermic exchange reaction, $\mathrm{H}_{2} \mathrm{D}^{+}+$ $\mathrm{H}_{2} \rightarrow \mathrm{H}_{3}^{+}+\mathrm{HD}$, becomes effective and dominates in the destruction of deuterated $\mathrm{H}_{3}^{+}$at higher temperatures. Since $\mathrm{H}_{2}$ is very abundant, the ratio of $\mathrm{H}_{2} \mathrm{D}^{+}$to $\mathrm{H}_{3}^{+}$rapidly reaches its equilibrium value, which is $\sim 1.3 \times 10^{-5}(T / 300 \mathrm{~K})^{-0.8} \exp (-230 \mathrm{~K} / T)$, i.e. $4 \times 10^{-3}$ and $3 \times 10^{-4}$ at $30 \mathrm{~K}$ and $50 \mathrm{~K}$, respectively (Millar. Bennet \& Herbst 1989). Hence $\mathrm{H}_{2} \mathrm{D}^{+}$and $\mathrm{HD}_{2}^{+}$may be good observational temperature probes in low- to intermediate-temperature regions $(\lesssim 50 \mathrm{~K})$ of the core.

Neutral species have high D/H ratios even in warm regions. These neutral species, such as $\mathrm{NH}_{3}, \mathrm{CH}_{4}$, and $\mathrm{CH}_{3} \mathrm{OH}$, are formed more efficiently via grain-surface reactions than by gas-phase reactions in the cold era. Inside the sublimation radii, the abundances of the sublimates overwhelm those of the products of gas-phase reactions. The icy material is highly deuterated by surface reactions with $\mathrm{D}$ atoms as described above, although the D/H ratios inside the sublimation radii are lower than the ratios of their gaseous counterparts in the $\mathrm{CO}$ depletion zone, since ices are formed and accumulated throughout the cold era, 
including the time before significant $\mathrm{CO}$ depletion occurs. For the same reason, the D/H ratios of the sublimates (i.e. inside the sublimation radii) are higher in earlier stages only by a factor of a few. The $\mathrm{D} / \mathrm{H}$ ratio of these gaseous neutral species is constant down to $r=2.5 \mathrm{AU}$, since their destruction time scales are longer than a free-fall time scale.

At $t=9.3 \times 10^{4} \mathrm{yr}$, various complex organics are formed via both gas-phase and grainsurface reactions; various carbon chains and large organics are formed via WCCC at $T>25$ $\mathrm{K}$ and via radical-radical surface reactions at $T \gtrsim 30 \mathrm{~K}$, respectively. Their $\mathrm{D} / \mathrm{H}$ ratios are high, mainly because they are formed from molecules with high $\mathrm{D} / \mathrm{H}$ ratios. As an example, methyl formate $\left(\mathrm{HCOOCH}_{3}\right)$ can be formed by the surface radical-radical reaction between the radicals $\mathrm{HCO}$ and $\mathrm{CH}_{3} \mathrm{O}$. Since both $\mathrm{HCO}$ and $\mathrm{CH}_{3} \mathrm{O}$ have high D/H ratios, $\mathrm{DCOOCH}_{3}$ and $\mathrm{HCOOCH}_{2} \mathrm{D}$ are also formed. The $\mathrm{HCOOCH}_{2} \mathrm{D}$ is three-times more abundant than $\mathrm{DCOOCH}_{3}$, since we assume the statistical branching rather than following the position of $\mathrm{D}$ in each reaction.

It is well known that the exchange reactions of $\mathrm{CH}_{3}^{+}+\mathrm{HD}$ and $\mathrm{C}_{2} \mathrm{H}_{2}^{+}+\mathrm{HD}$ are more exothermic than $\mathrm{H}_{3}^{+}+\mathrm{HD}$, and thus can be effective even at relatively warm temperatures (several tens of $\mathrm{K}$ ). However, they do not much contribute to the deuteration of WCCC species in our model, probably because carbon chains and $\mathrm{CH}_{4}$ already have high $\mathrm{D} / \mathrm{H}$ ratios when the fluid parcels enter the warm region, and because the duration of the warm temperature (around $30 \mathrm{~K}$ ) is short. The $\mathrm{D} / \mathrm{H}$ ratios of carbon-chain species change little, even if we neglect these two exchange reactions.

The exchange reaction of $\mathrm{OH}+\mathrm{D} \rightarrow \mathrm{OD}+\mathrm{H}$, on the other hand, has an even higher exothermicity $(\Delta E / k \sim 810 \mathrm{~K})$, and significantly deuterates $\mathrm{OH}$ at $t=9.3 \times 10^{4} \mathrm{yr}$ at radii of $100-1000 \mathrm{AU}$, where $\mathrm{HCOOH}$ has a higher $\mathrm{D} / \mathrm{H}$ ratio than other organic species due to the reaction of sublimated $\mathrm{H}_{2} \mathrm{CO}$ with $\mathrm{OD}$.

Radial distributions of assorted multiply-deuterated species are shown in Figure 4, together with mono-deuterated and normal isotopologues. The abundance ratios of multi- to mono-deuterated species are mainly determined statistically, e.g. $\mathrm{XD}_{2} / \mathrm{XHD} \sim \mathrm{XHD} / \mathrm{XH}_{2}$. For $\mathrm{CH}_{3} \mathrm{OH}$ and $\mathrm{H}_{2} \mathrm{CO}$, however, the multi- to mono ratio is lower than the mono-deuterated to normal isotope ratio. This effect could be due to the activation barriers in their formation path (i.e. in the reactions $\mathrm{H}+\mathrm{CO}$ and $\mathrm{H}+\mathrm{H}_{2} \mathrm{CO}$ ); the tunneling rate through the activation barrier is lower for heavier isotopomers (see discussion in $\S 4.3$ ). For $\mathrm{NH}_{3}$ and $\mathrm{CH}_{4}$, on the other hand, the multi-deuterated species are more abundant than expected from their mono-deuterated to normal isotope ratio. Although they are mainly formed on grain surfaces, gas-phase formation also contributes. In the gas-phase, multiply-deuterated $\mathrm{NH}_{3}$ and $\mathrm{CH}_{4}$ are efficiently formed in the $\mathrm{CO}$ depletion zone. Since the abundances of multiply-deuterated species are generally low, the efficient formation in the CO depletion 
zone effectively increases its abundance relative to the normal isotope. At $t=9.3 \times 10^{4} \mathrm{yr}$, multi-deuterated $\mathrm{NH}_{3}$ and $\mathrm{CH}_{4}$ are much less abundant than in early stages, because the CO depletion is much less significant.

In our model, we do not consider the layered structure of ice mantles. In reality, ice mantles have layered structures and the ices with the highest D/H ratios, which are formed at the latest stage of the cold prestellar phase, would lie on the very surface layer of the ice mantle, and would be the first to be sublimated. This effect creates radial gradients of $\mathrm{D} / \mathrm{H}$ ratios. For example, the $\mathrm{CH}_{4}$ formed in the $\mathrm{CO}$ depletion zone would have a higher D/H ratio than that formed in the early molecular cloud stage. Since the $\mathrm{CH}_{4}$ formed in the early stage would coexist within $\mathrm{H}_{2} \mathrm{O}$ ice, mainly the highly deuterated $\mathrm{CH}_{4}$ (in the surface layers) would be sublimated at $T \sim 25 \mathrm{~K}$; e.g. at $t=4.3 \times 10^{2}$ yr the gaseous $\mathrm{CH}_{4}$ abundance would be lower and $\mathrm{CH}_{3} \mathrm{D} / \mathrm{CH}_{4}$ ratio would higher in $r=10-100$ AU than shown in Figure 3 , Once the temperature reaches $T \gtrsim 150 \mathrm{~K}$, however, the dominant ice component, $\mathrm{H}_{2} \mathrm{O}$ ice, sublimates together with other species formed in the early stages of molecular clouds. Then the abundance and $\mathrm{D} / \mathrm{H}$ ratio of $\mathrm{CH}_{4}$, for example, would be the same as shown in Figure 3 inside $\sim 10 \mathrm{AU}$ at $t=4.3 \times 10^{2} \mathrm{yr}$.

\section{Discussion}

\subsection{Effect of $2 \mathrm{D}$ structure}

So far, we have adopted a spherically-symmetric model of star-forming cores. In the spherical model, fluid parcels fall to the central star over a short time scale. But in reality, dense cores are rotating with $\omega \sim 10^{-14} \mathrm{~s}^{-1}$, and the spherical symmetry is broken in the central regions as the collapse proceeds. Because of angular-momentum conservation, the centrifugal force increases for infalling fluid parcels and eventually balances the gravity at the centrifugal radius, which is given by the expression

$$
r_{\text {cent }}=\frac{\left(r^{2} \omega\right)_{\text {init }}^{2}}{G M} \sim 100\left(\frac{r}{0.07 \mathrm{pc}}\right)^{4}\left(\frac{\omega}{1 \times 10^{-14} \mathrm{~s}^{-1}}\right)^{2}\left(\frac{1 M_{\odot}}{M}\right) \mathrm{AU}
$$

to form a circumstellar disk, where $r$ and $M$ are the size and mass of the initial dense core, respectively. The result is that the fluid parcels stay in warm dense regions for a longer time scale than occurs in the spherical model.

In order to investigate the chemistry in this prolonged warm phase, we have performed a pseudo-time-dependent model (i.e., fixed density and temperature). For the initial abundances, we adopt the molecular abundances at $30 \mathrm{AU}$ in the final step of the spherical 
collapse model $\left(t=9.3 \times 10^{4} \mathrm{yr}\right)$, considering that the typical centrifugal radius is $\sim 100 \mathrm{AU}$ and that molecular abundances at 2.5 - $100 \mathrm{AU}$ are almost constant in the collapse model at $9.3 \times 10^{4} \mathrm{yr}$. All ices have already evaporated to the gas phase in this initial abundances (Figure 2).

Figure 5 shows the temporal variation of molecular abundances at $T=40 \mathrm{~K}(a-b)$ and $T=150 \mathrm{~K}(c-d)$. We choose these temperatures because grain-surface reactions would be active at $T=40 \mathrm{~K}$, while gas-phase reactions would dominate at $T=150 \mathrm{~K}$. The density is set to be $n_{\mathrm{H}}=1.487 \times 10^{8} \mathrm{~cm}^{-3}$, which is the density at $r=30 \mathrm{AU}$ in the $1 \mathrm{D}$ model. These parameter choices are rather arbitrary, but there should be regions with these densities and temperatures in the disks, because a real disk has density and temperature gradients in both the vertical and radial directions. We simply assume that the infalling fluid parcel reaches and stays in such regions in the disk. We also performed models with higher $(\times 10)$ and lower $(\times 0.1)$ densities, but the results are qualitatively the same; the timescale of the chemical evolution described below depends only slightly on the density (Nomura et al. 2009). At $T=40 \mathrm{~K}, \mathrm{C}_{2} \mathrm{H}_{6}$ increases and becomes the dominant carbon chain species. It is formed via grain-surface hydrogenation of $\mathrm{C}_{2} \mathrm{H}_{4}$, which is produced by the gas-phase reaction of $\mathrm{CH}+$ $\mathrm{CH}_{4}$. It should be noted that $\mathrm{C}_{2} \mathrm{H}_{4}\left(E_{\text {ads }}=3487 \mathrm{~K}\right.$ in our model; Garrod \& Herbst (2006) can marginally freeze onto grains, while $\mathrm{CH}\left(E_{\text {ads }}=925 \mathrm{~K}\right)$ and $\mathrm{CH}_{4}\left(E_{\text {ads }}=1300 \mathrm{~K}\right)$ are easily desorbed at $40 \mathrm{~K}$; adsorption of $\mathrm{C}_{2} \mathrm{H}_{4}$ onto the grain surface works as a sink in the gas-phase chemical network. Similarly, $\mathrm{C}_{7} \mathrm{H}_{4}$ ice $\left(E_{\mathrm{ads}}=7487 \mathrm{~K}\right)$ is the dominant carbon chain at $t=10^{6} \mathrm{yr}$ at $150 \mathrm{~K}$. Since the sublimation temperature of $\mathrm{C}_{7} \mathrm{H}_{4}$ is close to 150 $\mathrm{K}$, it depletes onto grains before additional carbon-chain growth in the gas phase at high densities, while hydrogenation on grain surface is inefficient at this high temperature.

The $\mathrm{D} / \mathrm{H}$ ratios of the carbon chains remain high at $T=40 \mathrm{~K}$, since the mother molecules (e.g. $\mathrm{CH}_{4}$ ) are highly deuterated in the cold phase. Also, the exothermic exchange reactions $\mathrm{CH}_{3}^{+}+\mathrm{HD}$ and $\mathrm{C}_{2} \mathrm{H}_{2}^{+}+\mathrm{HD}$, which have higher exothermicities than $\mathrm{H}_{3}^{+}+\mathrm{HD}$, contribute to keep the $\mathrm{D} / \mathrm{H}$ ratios high. At $T=150 \mathrm{~K}$, on the other hand, the $\mathrm{D} / \mathrm{H}$ ratio of carbon-chain species decreases gradually, because the endothermic exchange reactions (e.g. $\mathrm{CH}_{2} \mathrm{D}^{+}+\mathrm{H}_{2}$ and $\mathrm{C}_{2} \mathrm{HD}^{+}+\mathrm{H}_{2}$ ) can now proceed efficiently. It is interesting that species that undergo direct exchange reactions with $\mathrm{HD}$, such as $\mathrm{H}_{3}^{+}$and $\mathrm{C}_{2} \mathrm{H}_{2}^{+}$, work as a source of D/H enhancement in the network at low temperatures, but easily lose their own D enhancements at high temperature.

Among complex organic species, the $\mathrm{HCOOCH}_{3}$ abundance increases from $1.6 \times 10^{-9}$ (in the gas phase) to $\sim 10^{-7}$ (in the ice) via the grain-surface reaction of $\mathrm{HCO}+\mathrm{CH}_{3} \mathrm{O}$ in a few times $10^{4}$ yr at $T=40 \mathrm{~K}$, while the abundances of other complex species do not change significantly within this timescale. At $T=150 \mathrm{~K}$, on the other hand, a significant amount 
of $\mathrm{CH}_{3} \mathrm{OCH}_{3}$ is formed in the gas phase from $\mathrm{CH}_{3} \mathrm{OH}$ in $\sim 10^{5} \mathrm{yr}$. In spite of the formation at high temperatures, the $\mathrm{D} / \mathrm{H}$ ratios of $\mathrm{CH}_{3} \mathrm{OCH}_{3}$ is high, because the mother molecule, $\mathrm{CH}_{3} \mathrm{OH}$, is highly deuterated in the cold phase. At $t \gtrsim 10^{5} \mathrm{yr}$, complex organic species are destroyed via protonation and subsequent dissociative recombinations.

Another important feature missing in our spherical model is outflows. It creates a cavity in the envelope, through which the UV and X-ray from the protostar may escape. Photodissociation and photoionization play important roles near the cavity wall (Visser et al. 2011), or the surface of the circumstellar disks, if the envelope mass is small. The fraction of the mass going through such PDR-like regions is, however, rather limited: $\lesssim$ several \% (Visser et al. 2011). Our 1D model would be a reasonable approximation for the rest of the envelope regions, relocated from the outflow cavity.

\subsection{Comparison with observations}

Table 1 lists estimated abundances with respect to $\mathrm{H}_{2}$ from observations of oxygencontaining organic species in the protostellar core IRAS16293-2422. We distinguish the observations by single-dish telescopes and interferometers. IRAS16293-2422 is a binary system; interferometric observations distinguish the two sources IRAS16293A and IRAS16293B, while single-dish observations integrate the emission from the whole system. As can be seen from the diverse results, it is not straightforward to estimate molecular abundances in protostellar cores, because the cores have temperature and density gradients along the line of sight, and because the high-temperature regions, in which these complex organic species sublime, are mostly spatially unresolved even with the interferometer observations. Abundance estimates therefore vary significantly depending on the assumed temperature and density structure of the core.

It is noteworthy that interferometric observations tend to derive lower abundances of

large complex molecules than single dish observations corrected for beam dilution. Öberg et al. (2011) also noted such trends, and pointed out that cold extended component contributes significantly to the single dish observation. Thanks to the higher spatial resolution, interferometric observations could derive more reliable abundances in the central hot $(\gtrsim 100 \mathrm{~K})$ regions.

Table 1 also lists the abundances in our model near the center $(r=30 \mathrm{AU})$ of the protostellar core at $t=9.3 \times 10^{4} \mathrm{yr}$ and in our pseudo-time-dependent model with $T=40$ and $150 \mathrm{~K}$ at $t=10^{5} \mathrm{yr}$ from the start of the "disk". The core radius of $30 \mathrm{AU}$ is arbitrary, because the molecular abundances inside $\sim 100$ AU are almost constant due to rapid infall; 
it simply represents the abundances at $T \gtrsim 100 \mathrm{~K}$. As for the pseuo-time-dependent model at $T=40 \mathrm{~K}$, we list the ice abundances of complex organic species, assuming that they eventually accrete to smaller radii to be sublimated to the gas phase. As can be seen, the abundances of $\mathrm{H}_{2} \mathrm{CO}$ and $\mathrm{CH}_{3} \mathrm{OH}$ are higher in our core model than derived from observation. In the pseudo-time-dependent models, on the other hand, the $\mathrm{H}_{2} \mathrm{CO}$ abundance is far too low at both temperatures at the time used, while the methanol abundance for the $150 \mathrm{~K}$ model is in good agreement with observation. Note though that the abundances of $\mathrm{H}_{2} \mathrm{CO}$ and $\mathrm{CH}_{3} \mathrm{OH}$ are time-dependent; these species decrease with time at $150 \mathrm{~K}$ (Figure 5). We note that the spatial extent of the $\mathrm{CH}_{3} \mathrm{OH}$ sublimation zone (i.e. $T \gtrsim 100 \mathrm{~K}$ ) in the protostellar core is $\sim 100 \mathrm{AU}$ (Schöier et al. 2002), which is comparable to the typical centrifugal radius of dense cores. Therefore $\mathrm{CH}_{3} \mathrm{OH}$ can be partially destroyed in the prolonged warm temperature caused by rotational support. It is noteworthy that a recent ALMA observation of methyl formate revealed a velocity gradient which is consistent with rotating gas around IRAS16293A, although it is not ruled out that the velocity gradient could originate in two unresolved velocity components such as occur in a close binary (Pineda et al. 2012). Another explanation for the disagreement of $\mathrm{CH}_{3} \mathrm{OH}$ and $\mathrm{H}_{2} \mathrm{CO}$ abundances between our core model and the observation might be that these abundances are underestimated by observations. Observational constraints of molecule abundances in the warmer regions are uncertain because of beam dilution effects, the presence of multiple systems, and the possible presence of cavities and outflows. Indeed, interferometric observations which distinguish the two protostars in IRAS16293 system gives higher abundances of $\mathrm{CH}_{3} \mathrm{OH}$ than single dish observations.

Concerning the other molecules in the table, the model abundances for $\mathrm{HCOOCH}_{3}$, $\mathrm{HCOOH}$, and $\mathrm{CH}_{3} \mathrm{CN}$ in the core model are within the range of the estimated abundance from the observations. $\mathrm{CH}_{3} \mathrm{OCH}_{3}$, on the other hand, is significantly underestimated in our core model. In the pseudo-time-dependent model at $150 \mathrm{~K}$, however, the $\mathrm{CH}_{3} \mathrm{OCH}_{3}$ abundance increases with time and agrees with the observation at $t \sim 10^{5} \mathrm{yr}$. It is formed by the recombination of $\mathrm{CH}_{3} \mathrm{OCH}_{4}^{+}$in the gas phase. We note that the branching ratio for producing $\mathrm{CH}_{3} \mathrm{OCH}_{3}$ in the recombination is only $5 \%$ in our model. If we assume a higher branching ratio, $\mathrm{CH}_{3} \mathrm{OCH}_{3}$ becomes more abundant. Abundances of other species in the pseudo-time dependent model at $10^{5} \mathrm{yr}$ are also consistent with observations, except for $\mathrm{H}_{2} \mathrm{CO}$. This may indicate that the gases in the central hot region of IRAS16293 may have experienced prolonged warm temperature chemistry compared with the spherical infall model due to partial rotational support. Observations with higher angular resolution are highly desirable for more detailed comparison.

Our core and pseudo-time-dependent models can also be applied to carbon-chain species. Sakai et al. (2010) observed carbon chains towards L1527, and showed that the intensity 
distribution of $\mathrm{C}_{3} \mathrm{H}_{2}\left(4_{3,2}-4_{2,3}\right)$ is well reproduced, if its abundance jumps from $2.7 \times 10^{-9}$ to $2.7 \times 10^{-8}$ in the temperature range $T=23-29 \mathrm{~K}$. These peak abundances and temperature ranges are in reasonable agreement with our core model at $t=9.3 \times 10^{4}$ yr and $r \sim 10^{3}$ AU. Sakai et al. (2010) found that $\mathrm{C}_{2} \mathrm{H}, \mathrm{C}_{4} \mathrm{H}$, and $\mathrm{C}_{3} \mathrm{H}_{2}$ have a slight dip at the central region. In our spherical model, the central holes of the $\mathrm{C}_{2} \mathrm{H}$ and $\mathrm{C}_{4} \mathrm{H}$ abundances are reproduced, but $\mathrm{C}_{3} \mathrm{H}_{2}$ increases inwards, as discussed earlier. In the pseudo-time-dependent model at 40 $\mathrm{K}, \mathrm{C}_{3} \mathrm{H}_{2}$ is quickly depleted onto the grains, just like gaseous $\mathrm{C}_{2} \mathrm{H}_{6}$ in Figure 5 . The central dip of $\mathrm{C}_{3} \mathrm{H}_{2}$ thus might be reproduced, if the central region is dominated by the cold disk material rather than a warm infalling envelope. It is possible that the disk midplane is much cooler than the envelope because of the higher optical depth.

Let us now move on to deuterated species. Table 2 lists the estimated molecular D/H ratios in various protostellar cores and our model $\mathrm{D} / \mathrm{H}$ ratios. As in Table 1, we list model $\mathrm{D} / \mathrm{H}$ ratios at $t=9.3 \times 10^{4}$ yr of the protostellar core at $r=30 \mathrm{AU}$, except for $\mathrm{C}_{4} \mathrm{H}$, and in our pseudo-time-dependent models. For the $\mathrm{C}_{4} \mathrm{D} / \mathrm{C}_{4} \mathrm{H}$ ratio in the core model, we list the ratio at $r=250 \mathrm{AU}$, where $\mathrm{C}_{4} \mathrm{H}$ abundance reaches its maximum. Our $\mathrm{D} / \mathrm{H}$ ratios for the carbon chain species $\mathrm{C}_{4} \mathrm{H}$ and $\mathrm{C}_{3} \mathrm{H}_{2}$, and for $\mathrm{NH}_{3}$, are in reasonable agreement with the observations of L1527. On the other hand, the observed $\mathrm{D} / \mathrm{H}$ ratios of $\mathrm{NH}_{3}$ in $\mathrm{NGC1333}$ and $\mathrm{H}_{2} \mathrm{CO}$ in several protostellar cores are much higher than in our models (either core or disk). It should be noted, however, that in our core model these ratios are higher in the central warm region at early stages (e.g. $t=4.3 \times 10^{2} \mathrm{yr}$ ) and in the $\mathrm{CO}$ depletion zone than listed in Table 3 (Figure 4). The model $\mathrm{D} / \mathrm{H}$ ratios vary significantly depending on the evolutionary stage of the core, and the size of the warm regions, while the observational $\mathrm{D} / \mathrm{H}$ ratios thus depend on beam size (whether the beam traces mainly the cold component or the central warm regions). Such dependence also explains the variety of observed $\mathrm{D} / \mathrm{H}$ ratios towards protostars.

The $\mathrm{CH}_{2} \mathrm{DOH} / \mathrm{CH}_{3} \mathrm{OH}$ ratio reaches a high of 0.65 in the observations, while it is only a few per cent in our models. The comparison is even worse for $\mathrm{CHD}_{2} \mathrm{OH} / \mathrm{CH}_{3} \mathrm{OH}$. Unlike $\mathrm{NH}_{3}$ and $\mathrm{H}_{2} \mathrm{CO}, \mathrm{CH}_{3} \mathrm{OH}$ is not formed in the gas phase, and its gaseous abundance is very low in the $\mathrm{CO}$ depletion zone. Hence it is unlikely that the high $\mathrm{CH}_{2} \mathrm{DOH} / \mathrm{CH}_{3} \mathrm{OH}$ ratio originates in the $\mathrm{CO}$ depletion zone. In our model, $\mathrm{CH}_{2} \mathrm{DOH}$ is formed by the addition of $\mathrm{D}$ during the hydrogenation of $\mathrm{CO}$ on grain surfaces; we have not included substitution reactions of $\mathrm{H}_{2} \mathrm{CO}+\mathrm{D}$, or the abstraction reaction $\mathrm{CH}_{3} \mathrm{OH}+\mathrm{D}$, which are observed in laboratory experiments (Nagaoka et al. 2005, 2007; Hidaka et al. 2009). Taquet. Ceccarelli \& Kahane (2012) recently performed gas-grain chemical models at constant density and temperatures, and showed that these reactions are crucial to reproduce the observed $\mathrm{D} / \mathrm{H}$ ratios of $\mathrm{H}_{2} \mathrm{CO}$ and $\mathrm{CH}_{3} \mathrm{OH}$. In the following subsection $(\S 4.3)$, we add the substitution and abstraction reactions on grain surfaces to our network to see how strongly the $\mathrm{CH}_{2} \mathrm{DOH} / \mathrm{CH}_{3} \mathrm{OH}$ abun- 
dance ratio is enhanced.

The $\mathrm{D} / \mathrm{H}$ ratio of water ice in the envelope at $t=9.3 \times 10^{4} \mathrm{yr}$ is $\sim 1 \%$, which is marginally consistent with the upper limits obtained towards low-mass protostars (Parise et al. 2003). Recently gaseous HDO has also been intensively searched for towards protostellar cores using ground-based single-dish telescopes and the Herschel Space Observatory. Table 3 lists the estimated $\mathrm{HDO}$ abundance and $\mathrm{HDO} / \mathrm{H}_{2} \mathrm{O}$ ratio in the gas phase in the hot corino region $(T \geq 100 \mathrm{~K})$ and outer envelope $(T \leq 100 \mathrm{~K})$ towards NGC 1333-IRAS2A and IRAS16293 (Liu et al. 2011; Coutens et al. 2012). Towards IRAS16293, an absorbing layer is added in front of the envelope to account for the observed absorption lines. Our model $\mathrm{HDO} / \mathrm{H}_{2} \mathrm{O}$ ratio in the hot region is consistent with the observations, while our HDO abundance is higher by an order of magnitude (see panel $(a)$ of $t=9.3 \times 10^{4} \mathrm{yr}$ in Figure 3 ). We note that the $\mathrm{HDO}$ (and $\mathrm{H}_{2} \mathrm{O}$ ) abundance could be underestimated in observations, just like $\mathrm{CH}_{3} \mathrm{OH}$ and $\mathrm{H}_{2} \mathrm{CO}$, because of the complex (and poorly-constrained) structures of the source such as the presence of multiple systems (IRAS16293), and the possible presence of cavities, outflows and disks. In addition to the targets listed in Table 3, Jørgensen \& van Dishoeck (2010) searched for gaseous HDO towards NGC1333-IRAS4B using the Sub-Millimeter Array (SMA). The line was not detected, which constrains the $\mathrm{HDO} / \mathrm{H}_{2} \mathrm{O}$ ratio $\leq 6 \times 10^{-4}$ in the central hot region $(\sim 50 \mathrm{AU})$. This upper limit is much lower than predicted in our model. Since gaseous HDO in the central region originate in ice formed in the cold era, the variation of the $\mathrm{HDO} / \mathrm{H}_{2} \mathrm{O}$ ratio among protostars could originates in the temperature variation in the very early stages of molecular clouds or translucent clouds (Cazaux et al. 2011).

In the outer envelope, our $\mathrm{HDO}$ abundance and $\mathrm{HDO} / \mathrm{H}_{2} \mathrm{O}$ ratio at $\gtrsim 10^{3} \mathrm{AU}$ at $t=$ $9.3 \times 10^{4} \mathrm{yr}$ are in reasonable agreement with NGC1333-IRAS2A and absorbing layer of IRAS16293 (Figure 3). But the observed values in the envelope of IRAS16293 is lower than in our model at $t=9.3 \times 10^{4} \mathrm{yr}$. Since the molecular column densities in the envelope are lower in earlier stages, a model at slightly earlier stage than $t=9.3 \times 10^{4}$ yr might be more consistent with the envelope HDO abundance in IRAS16293. It should also be noted that in our model at $t=9.3 \times 10^{4} \mathrm{yr}, \mathrm{HDO} / \mathrm{H}_{2} \mathrm{O}$ ratio is close to unity around $500 \mathrm{AU}$, which is a part of the outer envelope (Figure 3). This high $\mathrm{HDO} / \mathrm{H}_{2} \mathrm{O}$ ratio is caused by the exothermic exchange reaction of $\mathrm{OH}+\mathrm{D}$; the rate coefficient is set to $k=1.3 \times 10^{-10} \mathrm{~cm}^{3} \mathrm{~s}^{-1}$ (Millar. Bennet \& Herbst 1989), which is consistent with a detailed theoretical calculation by Atahan et al. (2005) $\left(1.5 \times 10^{-10} \mathrm{~cm}^{3} \mathrm{~s}^{-1}\right.$ at $\left.50 \mathrm{~K}\right)$. Such local $\mathrm{HDO} / \mathrm{H}_{2} \mathrm{O}$ enhancement has not been observed thus far. Although the effect of the $\mathrm{OH}+\mathrm{D}$ reaction seems to be transient and local in our model, a further search for such enhancements could constrain O-chemistry in the protostellar envelope. 
Table 1: Observed and Model Gas-Phase Molecular Abundances in IRAS 16293-2422

\begin{tabular}{lccccc}
\hline Species & \multicolumn{2}{c}{ IRAS $16293-2422$} & \multicolumn{3}{c}{ model $^{\mathrm{a}}$} \\
& single dish & interferometer & core & $40 \mathrm{~K}$ & $150 \mathrm{~K}$ \\
\hline $\mathrm{H}_{2} \mathrm{CO}$ & $1.0(-7)^{\mathrm{b}}$ & $1.1(-7)^{\mathrm{c}}$ & $1.1(-5)$ & $5.4(-10)^{\mathrm{d}}$ & $1.1(-11)$ \\
$\mathrm{CH}_{3} \mathrm{OH}$ & $1.0(-7)^{\mathrm{e}}$ & $9.4(-8)^{\mathrm{c}}, 6.8(-7)^{\mathrm{f}}, 3.1(-7)^{\mathrm{g}}$ & $3.6(-6)$ & $2.9(-6)^{\mathrm{d}}$ & $5.2(-7)$ \\
$\mathrm{HCOOCH}_{3}$ & $4.0(-7)^{\mathrm{h}}$ & $7.5(-9)^{\mathrm{i}}, 4.3(-9)^{\mathrm{f}}, 2.6(-9)^{\mathrm{g}}$ & $3.1(-9)$ & $1.7(-7)^{\mathrm{d}}$ & $8.8(-10)$ \\
& \multicolumn{3}{c}{} & & \\
$\mathrm{HCOOH}$ & $6.2(-8)^{\mathrm{h}}$ & $4.9(-10)^{\mathrm{j}},>1.2(-8)^{\mathrm{k}}$ & & \\
$\mathrm{CH}_{3} \mathrm{OCH}$ & & $2.5(-9)^{\mathrm{k}}$ & $1.1(-8)$ & $3.3(-8)^{\mathrm{d}}$ & $1.2(-8)$ \\
$\mathrm{CH}_{3} \mathrm{CN}$ & $2.4(-7)^{\mathrm{h}}$ & $7.6(-8)^{\mathrm{c}}$ & $1.2(-10)$ & $3.9(-10)^{\mathrm{d}}$ & $3.3(-8)$ \\
\hline
\end{tabular}

${ }^{a}$ Molecular abundances at $r=30 \mathrm{AU}$ and $t=9.3 \times 10^{4} \mathrm{yr}$ in the protostellar core model, and in the pseudo-time-dependent model of $T=40 \mathrm{~K}$ and $150 \mathrm{~K}$ at $t=1 \times 10^{5}$ from the start of the "disk".

Maret et al. 2004)

Chandler et al. (2005)

$d_{\text {ice abundance }}$

Maret et al. (2005)

Kuan et al. (2004), IRAS16293A

9 Kuan et al. (2004), IRAS16293B

h Cazaux et al. (2003)

${ }^{i}$ IRAS16293 A (Bottinelli et al. 2004)

${ }^{j}$ IRAS16293 B (Bottinelli et al. 2004)

${ }^{k}$ Remiiian \& Hollis (2006)

Schöier et al. (2002) 
Table 2: Molecular D/H ratios in protostellar cores

\begin{tabular}{|c|c|c|c|c|c|c|}
\hline \multirow[t]{2}{*}{ Species } & \multirow[t]{2}{*}{$\mathrm{D} / \mathrm{H}$ ratio } & \multirow[t]{2}{*}{ source } & \multicolumn{3}{|c|}{ model $^{\mathrm{a}}$} & \multirow[t]{2}{*}{ reference } \\
\hline & & & core & $40 \mathrm{~K}$ & $150 \mathrm{~K}$ & \\
\hline \multirow[t]{2}{*}{$\mathrm{NH}_{2} \mathrm{D} / \mathrm{NH}_{3}$} & 0.28 & NGC1333 & 0.015 & $0.014^{\mathrm{b}}$ & 0.012 & Roueff et al. (2005) \\
\hline & 0.04 & L1527 & & & & Sakai et al. (2009) \\
\hline $\mathrm{ND}_{3} / \mathrm{NH}_{3}$ & $2.1 \times 10^{-4}$ & NGC1333 & $1.6 \times 10^{-6}$ & $1.5 \times 10^{-6 b}$ & $5.1 \times 10^{-7}$ & Roueff et al. (2005) \\
\hline $\mathrm{HDCO} / \mathrm{H}_{2} \mathrm{CO}$ & $0.094-1.7$ & 7 protostars & 0.026 & $0.013^{\mathrm{b}}$ & $-c$ & Parise et al. (2006) \\
\hline $\mathrm{D}_{2} \mathrm{CO} / \mathrm{H}_{2} \mathrm{CO}$ & $0.046-0.44$ & 7 protostars & $3.3 \times 10^{-5}$ & $2.9 \times 10^{-5 b}$ & $-c$ & Parise et al. (2006) \\
\hline \multirow[t]{2}{*}{$\mathrm{CH}_{2} \mathrm{DOH} / \mathrm{CH}_{3} \mathrm{OH}$} & $0.37-0.65$ & 4 protostars & 0.024 & $0.026^{\mathrm{b}}$ & 0.023 & Parise et al. (2006) \\
\hline & $\leq 0.030$ & L1527 & & & & Sakai et al. (2009) \\
\hline $\mathrm{CHD}_{2} \mathrm{OH} / \mathrm{CH}_{3} \mathrm{OH}$ & $0.074-0.25$ & 4 protostars & $1.7 \times 10^{-4}$ & $2.0 \times 10^{-4 b}$ & $1.5 \times 10^{-4}$ & Parise et al. (2006) \\
\hline $\mathrm{C}_{4} \mathrm{D} / \mathrm{C}_{4} \mathrm{H}$ & 0.018 & L1527 & $0.033^{\mathrm{d}}$ & $-\mathrm{c}$ & $-c$ & Sakai et al. (2009) \\
\hline $\mathrm{C}_{3} \mathrm{HD} / \mathrm{C}_{3} \mathrm{H}_{2}$ & 0.071 & L1527 & 0.033 & $0.016^{\mathrm{b}}$ & 0.0083 & Sakai et al. (2009) \\
\hline
\end{tabular}

${ }^{a}$ Molecular abundances at $r=30 \mathrm{AU}$ and $t=9.3 \times 10^{4} \mathrm{yr}$ in the protostellar core model, and in the pseudo-time-dependent model of $T=40 \mathrm{~K}$ and $150 \mathrm{~K}$ at $t=1 \times 10^{5}$ from the start of the "disk".

${ }^{b}$ ice abundance

${ }^{c} \mathrm{D} / \mathrm{H}$ ratio is not listed because the abundance of the molecule is too small compared with observations.

${ }^{d} \mathrm{D} / \mathrm{H}$ ratio at $r=250 \mathrm{AU}$, where $\mathrm{C}_{4} \mathrm{H}$ abundance reaches its maximum.

Table 3: $\mathrm{HDO}$ abundance and $\mathrm{HDO} / \mathrm{H} 2 \mathrm{O}$ ratio in the gas phase in protostellar cores

\begin{tabular}{lcccc}
\hline & hot $(T \geq 100 \mathrm{~K})$ & $\begin{array}{c}\text { regions } \\
\text { envelope }(T \leq 100 \mathrm{~K})\end{array}$ & absorbing & \\
\hline NGC1333-IRAS2A & & & Liu et al. $(2011)$ \\
HDO abundance & $6.6 \times 10^{-8}-1.0 \times 10^{-7}$ & $9 \times 10^{-11}-1.8 \times 10^{-9}$ & - & \\
$\mathrm{HDO} / \mathrm{H}_{2} \mathrm{O}$ & $\geq 1 \%$ & $0.9-18 \%$ & - & \\
IRAS16293-2422 & & & & Coutens et al. (2012) \\
$\mathrm{HDO}$ abundance & $1.7 \times 10^{-7}$ & $8 \times 10^{-11}$ & & \\
$\mathrm{HDO} / \mathrm{H}_{2} \mathrm{O}$ & $1.4-5.8 \%$ & $0.2-2.2 \%$ & $4.8 \%$ & \\
\hline
\end{tabular}




\subsection{Deuteration of $\mathrm{CH}_{3} \mathrm{OH}$ on grain surfaces}

The grain-surface reaction network for $\mathrm{H}_{2} \mathrm{CO}$ and $\mathrm{CH}_{3} \mathrm{OH}$ derived from laboratory experiments is summarized in Watanabe \& Kouchi (2008) and Hidaka et al. (2009). Most of the reactions are already included in our original gas-grain model, except for the substitution $\mathrm{H}_{2} \mathrm{CO}+\mathrm{D} \rightarrow \mathrm{HDCO}+\mathrm{H}$, abstraction $\mathrm{CH}_{3} \mathrm{OH}+\mathrm{D} \rightarrow \mathrm{CH}_{2} \mathrm{OH}+\mathrm{HD}$, and their deuterated analogues (Figure 6). Here we add these reactions to our model.

Laboratory experiments indicate that the abstraction reaction $\mathrm{CH}_{3} \mathrm{OH}+\mathrm{D}$ produces $\mathrm{CH}_{2} \mathrm{OH}$ but not $\mathrm{CH}_{3} \mathrm{O}$, so the subsequent hydrogenation reaction can form $\mathrm{CH}_{2} \mathrm{DOH}$ but not $\mathrm{CH}_{3} \mathrm{OD}$, which is in agreement with the observed low abundance of $\mathrm{CH}_{n} \mathrm{D}_{3-n} \mathrm{OD}$ compared with $\mathrm{CH}_{n} \mathrm{D}_{3-n} \mathrm{OH}$ (Parise et al. 2004; Nagaoka et al. 2005). In our original deuterated network model, however, we did not follow the position of the deuterium atoms in the reactions, and we did not fully distinguish the various possible isomers of radicals, such as $\mathrm{CH}_{2} \mathrm{DO}$, $\mathrm{CHDOH}$ and $\mathrm{CH}_{2} \mathrm{OD}$, which are produced in the sequence of deuterated methanol formation. Our model thus underestimates (dilutes) the isomer ratio $\mathrm{CH}_{n} \mathrm{D}_{3-n} \mathrm{OH} / \mathrm{CH}_{n} \mathrm{D}_{2-n} \mathrm{OD}$, although we distinguish isomers in the abstraction reactions if they are in our original species list. In spite of the imperfect distinction of the isomers, $\mathrm{CH}_{2} \mathrm{DOH}$ is more abundant than $\mathrm{CH}_{3} \mathrm{OD}$ in our model.

The activation barriers of the substitution and abstraction reactions have recently been investigated using theoretical calculations (e.g. Goumans 2011); however, the rate is determined not only by activation barrier height. Since the reactions proceed via tunneling at low temperatures, the tunneling mass and the shape (width) of the potential barrier are also important. Thus, the accurate evaluation of reaction rates from theoretical calculations is challenging. Rather than incorporate activation barriers from theoretical calculations, here

we utilize the rates derived from the laboratory experiments by Watanabe \& Kouchi (2008) and Hidaka et al. (2009), who estimated relative rate coefficients of various reactions to the rate of $\mathrm{H}+\mathrm{CO}$. In our model, rate coefficients for grain-surface reactions are proportional to a frequency factor times $\exp \left[-2 w / \hbar \sqrt{2 m E_{\text {act }}}\right]$, where $w$ is a width of the barrier, $m$ is the effective mass, and $E_{\text {act }}$ is the height of the barrier, if the reaction proceeds via tunneling. The width of the barrier $w$ is fixed to $1 \AA$. The effective mass is equal to the reduced mass of the reactants in a two-body addition reaction, but depends on the details of reaction for more complex reactions such as abstraction (Hidaka et al. 2009). Since our model includes various grain-surface reactions of which the details (such as potential energy surface) are not yet well understood, we use the reduced mass of the reactants as the effective mass for all grain-surface reactions. The activation barrier of the reaction $\mathrm{H}+\mathrm{CO}$ is set to $2500 \mathrm{~K}$ in our original model. Figure [6] shows reactions in our model and $E_{\text {act }}$ which we assume for each reaction in units of $\mathrm{K} ; E_{\text {act }}$ is determined so that the rate coefficients relative to $\mathrm{H}+$ 
CO become roughly consistent with Watanabe \& Kouchi (2008) and Hidaka et al. (2009). Reactions depicted by the solid arrows are included in our original model, while the dashed arrows are newly added reactions. In the original model, the activation barrier for D + CO is set to be the same as $\mathrm{H}+\mathrm{CO}(2500 \mathrm{~K})$; due to the larger effective mass, the rate coefficient of $\mathrm{D}+\mathrm{CO}$ is lower than that of $\mathrm{H}+\mathrm{CO}$ by several orders of magnitude. In the laboratory experiment, on the other hand, the relative rate coefficient of $\mathrm{D}+\mathrm{CO}$ is about $10 \%$ to $\mathrm{H}$ + CO. Hence we changed the barrier for D + CO to $1400 \mathrm{~K}$ in the model. Similarly the barrier for reaction of $\mathrm{D}$ atom with $\mathrm{H}_{2} \mathrm{CO}$ (and its deuterated isotopomers) are lowered. It should be noted that these values of $E_{\text {act }}$ are not necessarily equal to the activation barrier obtained from the quantum chemical calculations, since the rate of the tunneling sensitively depends on the shape of the potential and effective mass in reality, while we assume a simple rectangular barrier and adopt the reduced mass as the effective mass. We also note that the frequency factor is often replaced by accretion or desorption rates of $\mathrm{H}$ and $\mathrm{D}$ atoms in our calculation, because we adopt the modified rate.

Taquet, Ceccarelli \& Kahane (2012) calculated the D/H ratios of $\mathrm{H}_{2} \mathrm{CO}$ and $\mathrm{CH}_{3} \mathrm{OH}$ in pseudo-time-dependent models of $n_{\mathrm{H}}=10^{4}-5 \times 10^{6} \mathrm{~cm}^{-3} \mathrm{~cm}^{-3}$, including the substitution and abstraction reactions. Their grain-surface chemistry model is more sophisticated than ours; they consider multiple layers and cracks in the grain mantles. The rate coefficients are determined relative to the $\mathrm{H}+\mathrm{CO}$ rate, referring to Watanabe et al. (2010) and Hidaka et al. (2009). They showed that the D/H ratios reach higher values at higher densities; in the model with $n_{\mathrm{H}}=5 \times 10^{6} \mathrm{~cm}^{-3}$ and $T=10 \mathrm{~K}$, the $\mathrm{CH}_{2} \mathrm{DOH} / \mathrm{CH}_{3} \mathrm{OH}$ ratio exceeds unity at $t \gtrsim 10^{4} \mathrm{yr}$.

In order to compare our chemical network model with the work of Taquet, Ceccarelli \& Kahane (2012), we calculated a pseudo-time-dependent model with $n_{\mathrm{H}}=5 \times 10^{6} \mathrm{~cm}^{-3}$ and $T=10$ $\mathrm{K}$; Figure 7 shows the temporal variation of $\mathrm{H}_{2} \mathrm{CO}, \mathrm{CH}_{3} \mathrm{OH}$ and their deuterated isotopes in our model. The initial abundances are set by solving the network under molecular-cloud conditions $\left(n_{\mathrm{H}}=2 \times 10^{4} \mathrm{~cm}^{-3}, T=10 \mathrm{~K}\right)$ for $8 \times 10^{4} \mathrm{yr}$ in panel (a) and for $2 \times 10^{5} \mathrm{yr}$ in panel (b). The molecular D/H ratios become very high $0.1-1.0$ at several $10^{4}-10^{5} \mathrm{yr}$. The rise in the $\mathrm{D} / \mathrm{H}$ ratio is slower than in the model of Taquet, Ceccarelli \& Kahane (2012), in which the $\mathrm{HDCO} / \mathrm{H}_{2} \mathrm{CO}$ ratio exceeds 0.1 at $\sim 10^{3} \mathrm{yr}$ and the $\mathrm{CH}_{2} \mathrm{DOH} / \mathrm{CH}_{3} \mathrm{OH}$ ratio exceeds unity at $\sim 10^{4} \mathrm{yr}$. The slow rise of the $\mathrm{D} / \mathrm{H}$ ratio in our model would be mainly due to the initial abundances. Taquet, Ceccarelli \& Kahane (2012) calculate the atomic D/H ratio in steady state as a function of density, and adopt it as an initial abundance. The steady-state atomic D/H ratio is greater under higher-density conditions. Our initial abundances, on the other hand, are determined by the molecular-cloud calculation of $n_{\mathrm{H}}=2 \times 10^{4}$ $\mathrm{cm}^{-3}$. In our pseudo-time-dependent model of $n_{\mathrm{H}}=5 \times 10^{6} \mathrm{~cm}^{-3}$, the atomic $\mathrm{D} / \mathrm{H}$ ratio increases in $10^{4} \mathrm{yr}$ to reach the steady state. Initial abundances of $\mathrm{CO}, \mathrm{CH}_{3} \mathrm{OH}$ and $\mathrm{H}_{2} \mathrm{CO}$ 
would also be relevant; the temporal variation of the molecular $\mathrm{D} / \mathrm{H}$ ratio differs in panel (a) and (b). Although deuterated $\mathrm{H}_{2} \mathrm{CO}$ and $\mathrm{CH}_{3} \mathrm{OH}$ increase rapidly both in (a) and (b) at $t \gtrsim 10^{4} \mathrm{yr}$, when the atomic $\mathrm{D} / \mathrm{H}$ ratio reaches steady state, the molecular $\mathrm{D} / \mathrm{H}$ ratios do not reach unity in panel (b), due to the high abundance of normal isotopes in the initial conditions. The model of Taquet, Ceccarelli \& Kahane (2012), on the other hand, assumes that all carbon initially resides in $\mathrm{CO}$.

Having tested our chemical network against Taquet, Ceccarelli \& Kahane (2012), we now calculate molecular abundances and $\mathrm{D} / \mathrm{H}$ ratios including the substitution and abstraction reactions at $t=-5.6 \times 10^{2} \mathrm{yr}, 4.3 \times 10^{2} \mathrm{yr}$ and $9.3 \times 10^{4} \mathrm{yr}$ in our star-forming cores. The result is almost the same as in Figure 3; the $\mathrm{D} / \mathrm{H}$ ratio increases at most by a factor of a few. There are three possible reasons for this low $\mathrm{D} / \mathrm{H}$ ratio in spite of the newly-added reactions:

(i) Our model starts from a dense molecular cloud core. A significant amount of $\mathrm{CH}_{3} \mathrm{OH}$ and $\mathrm{H}_{2} \mathrm{CO}$ is already formed during the pre-collapse phase with $n_{\mathrm{H}} \lesssim 5 \times 10^{4} \mathrm{~cm}^{-3}$, during which the deuterium enrichment by the new reactions are not very efficient (Taquet, Ceccarelli \& Kahane 2012).

(ii) The period spent at high density $\left(\gtrsim 10^{6} \mathrm{~cm}^{-3}\right)$, in which the deuterium enrichment is efficient, is limited by the dynamical model. The temporal variation of density in the infalling fluid parcels that reach $R=2.5 \mathrm{AU}$ at $9.3 \times 10^{4} \mathrm{yr}$ is shown in Figure 1 . The parcel falls onto the protostar in several $10^{3}$ yrs after it enters the region where $n_{\mathrm{H}} \geq 10^{6} \mathrm{~cm}^{-3}$.

(iii) The duration of the high-density phase is longer in fluid parcels that reach the central region in earlier stages (e.g. $t=-5.6 \times 10^{2}$ yr and $4.3 \times 10^{2} \mathrm{yr}$ ); but the dust temperature for such parcels is mostly very low; it could reach $\sim 5 \mathrm{~K}$ (e.g. Keto \& Caselli 2010). Since we assume thermal hopping, even $\mathrm{H}$ and $\mathrm{D}$ atoms cannot efficiently migrate on grains.

Our model indicates that the $\mathrm{D} / \mathrm{H}$ ratios of $\mathrm{CH}_{3} \mathrm{OH}$ and $\mathrm{H}_{2} \mathrm{CO}$ in the star-forming core depend significantly on the dynamical model, the heat balance (e.g. cosmic-ray flux) inside the prestellar cores, and the efficiency of grain-surface migration of $\mathrm{H}$ and $\mathrm{D}$ atoms at very low temperature. For example, we found that the gaseous $\mathrm{CH}_{2} \mathrm{DOH} / \mathrm{CH}_{3} \mathrm{OH}$ ratio reaches $17 \%$ inside the sublimation radius $(r \leq 7.5 \mathrm{AU})$ at $t=4.3 \times 10^{2} \mathrm{yr}$, if we allow $\mathrm{H}$ and $\mathrm{D}$ atoms to migrate via tunneling. Recent laboratory work excludes the migration of $\mathrm{H}$ and D atoms via tunneling, but finds fast thermal hopping at $8 \mathrm{~K}$ (Watanabe et al. 2010). Considering the strong dependence of the hopping rate on temperature, thermal hopping of 
$\mathrm{H}$ and $\mathrm{D}$ atoms could still be inefficient at $5 \mathrm{~K}$. In such a case, the Eley-Rideal mechanism, which is not included in our model, could be important. We postpone further investigation of this issue to future work.

\section{Summary}

We have investigated molecular abundances and $\mathrm{D} / \mathrm{H}$ ratios in a star-forming core by adopting the $1 \mathrm{D}$ radiation hydrodynamics model of Masunaga \& Inutsuka (2000).

Spatial distributions of assorted molecules in the gas phase and ice mantle are reported for a core at a time $5.6 \times 10^{2}$ yr prior to protostar formation (at which the so-called first core is formed), and at $4.3 \times 10^{2} \mathrm{yr}$ and $9.3 \times 10^{4} \mathrm{yr}$ after the protostar is born. Significant depletion onto grains occurs in the early stages, at radii $10 \mathrm{AU} \lesssim r \lesssim 10^{3} \mathrm{AU}$ and $100 \mathrm{AU}$ $\lesssim r \lesssim 10^{3} \mathrm{AU}$, for times $t=-5.6 \times 10^{-2} \mathrm{yr}$ and $4.3 \times 10^{2} \mathrm{yr}$, respectively. As the core gets warmer, complex species, as well as simple species such as $\mathrm{NH}_{3}$, sublime from the grain surfaces near the core center. The abundances of these complex species - both in the gas phase and ice mantle - increase with time, while the variation is rather small for $\mathrm{CH}_{3} \mathrm{CN}$, which can be formed readily at low temperatures. Methanol, which can also be formed at low temperatures, decreases gradually as the core evolves. We find reasonable agreement with observation for our model abundances at $t=9.3 \times 10^{4} \mathrm{yr}$ and $T \gtrsim 100 \mathrm{~K}$, except for $\mathrm{CH}_{3} \mathrm{OCH}_{3}$, which is significantly under-abundant in our $1 \mathrm{D}$ core model.

Unsaturated carbon chains are formed from sublimed $\mathrm{CH}_{4}$ at $T \sim 25 \mathrm{~K}(r \sim 1000$ AU) at $t=9.3 \times 10^{4} \mathrm{yr}$. We show that the chemistry that forms these chains is indeed the WCCC mechanism developed by Sakai et al. (2008) to explain the chemistry in L1527 and IRAS15398-3359. Our model also reproduces the central dip for $\mathrm{C}_{2} \mathrm{H}$ and $\mathrm{C}_{4} \mathrm{H}$ observed by Sakai et al. (2010). In the earlier stages, however, the gas-phase formation of unsaturated carbon chains is much less efficient due to the higher density at the $\mathrm{CH}_{4}$ sublimation zone. In order for WCCC to be efficient, the gas density at the $\mathrm{CH}_{4}$ sublimation zone should be relatively low, so that $\mathrm{C}^{+}$, which is another ingredient for carbon chain formation, becomes abundant.

In the cold phase $(T \sim 10 \mathrm{~K})$, molecular $\mathrm{D} / \mathrm{H}$ ratios are enhanced by exothermic exchange reactions such as $\mathrm{H}_{2} \mathrm{D}^{+}+\mathrm{HD}$. We find very high molecular $\mathrm{D} / \mathrm{H}$ ratios (close to unity) for gas-phase species in the $\mathrm{CO}$ depletion zone, because $\mathrm{CO}$ is the main reaction partner of $\mathrm{H}_{2} \mathrm{D}^{+}$. In the central regions of the protostellar core, however, molecular $\mathrm{D} / \mathrm{H}$ ratios are mostly $\sim 10^{-2}$; they originate in the $\mathrm{D} / \mathrm{H}$ ratios formed on ice mantles, which are accumulated from the cloud core phase (without significant CO depletion) all the way 
through to the protostellar phase. At $t=9.3 \times 10^{4} \mathrm{yr}$, a significant amount of complex organic species and carbon chains are formed via grain-surface and gas-phase reactions at warm temperatures $(T \gtrsim 30 \mathrm{~K}$ for organic species and $T \gtrsim 25 \mathrm{~K}$ for carbon chains). Their $\mathrm{D} / \mathrm{H}$ ratios are $\sim 10^{-2}$, inheriting the $\mathrm{D} / \mathrm{H}$ ratio of their precursor molecules, and vary slightly among species. Exothermic exchange reactions are still active at temperatures of a few tens of Kelvin, although the rates of the backward reactions increase with temperature. $\mathrm{HCOOH}$ has a higher $\mathrm{D} / \mathrm{H}$ ratio than other complex organic species; it is formed by $\mathrm{H}_{2} \mathrm{CO}+\mathrm{OH}$, so that HCOOD can be formed from $\mathrm{H}_{2} \mathrm{CO}+\mathrm{OD}$ where $\mathrm{OH}$ is deuterated by the exchange reaction of $\mathrm{OH}+\mathrm{D}$. This exchange reaction also significantly enhances the $\mathrm{HDO} / \mathrm{H}_{2} \mathrm{O}$ ratio at $T \sim 50 \mathrm{~K}$.

While the observed molecular $\mathrm{D} / \mathrm{H}$ ratios in protostellar cores could be mostly explained by the combination of $\mathrm{D} / \mathrm{H}$ ratios in the hot-corino region (i.e. $T>100 \mathrm{~K}$ ) and $\mathrm{CO}$ depletion zone, the $\mathrm{D} / \mathrm{H}$ ratio of $\mathrm{CH}_{3} \mathrm{OH}$ are significantly underestimated in our model. Laboratory experiments (Nagaoka et al. 2005; Watanabe \& Kouchi 2008; Hidaka et al. 2009) found substitution and abstraction reactions on grain surfaces to deuterate $\mathrm{H}_{2} \mathrm{CO}$ and $\mathrm{CH}_{3} \mathrm{OH}$; we have added these reactions to our model. Although the $\mathrm{D} / \mathrm{H}$ ratios of $\mathrm{CH}_{3} \mathrm{OH}$ and $\mathrm{H}_{2} \mathrm{CO}$ can exceed unity at $t \gtrsim 10^{5} \mathrm{yr}$ in a pseudo-time-dependent model with a high density $\left(n_{\mathrm{H}}=5 \times 10^{6} \mathrm{~cm}^{-3}\right)$ and low temperature $(T=10 \mathrm{~K})$, the ratios increased only by a factor of a few in our protostellar core model. A significant amount of $\mathrm{CH}_{3} \mathrm{OH}$ ice is formed in the prestellar stage, in which the density is still too low for the newly-added reactions to be efficient. While the high density phase lasts longer in infalling fluid parcels in earlier evolutionary stage of a core, the temperature could be as low as $5 \mathrm{~K}$ there. Eley-Rideal mechanism, rather than Langmuir-Hinshelwood mechanism assumed in our model, could therefore be important. Further studies are needed on this issue.

Finally, in order to see how the molecular abundances evolve after fluid parcels land on the disk, we performed pseudo-time-dependent models at $T=40 \mathrm{~K}$ and $150 \mathrm{~K}$ starting from the molecular abundances set by the $1 \mathrm{D}$ collapse model. At $T=40 \mathrm{~K}$, a significant amount of $\mathrm{HCOOCH}_{3}$ ice is formed by the grain-surface reactions. $\mathrm{C}_{2} \mathrm{H}_{6}$ ice becomes abundant among carbon chains; it is formed via grain-surface hydrogenation of $\mathrm{C}_{2} \mathrm{H}_{4}$, which is produced by a gas-phase reaction. Since $\mathrm{C}_{2} \mathrm{H}_{4}$ can marginally freeze onto grains at $40 \mathrm{~K}$, its adsorption onto grains works as a sink in the gas-phase chemical reaction network. At $T=150 \mathrm{~K}, \mathrm{C}_{7} \mathrm{H}_{4}$ ice becomes the dominant carbon chain, and its $\mathrm{D} / \mathrm{H}$ ratio decreases with time at $t \gtrsim 10^{5} \mathrm{yr}$. Meanwhile, $\mathrm{CH}_{3} \mathrm{OCH}_{3}$ is efficiently formed in the high-temperature gas from the sublimed $\mathrm{CH}_{3} \mathrm{OH}$. It is noteworthy that $\mathrm{CH}_{3} \mathrm{OCH}_{3}$ has a high $\mathrm{D} / \mathrm{H}$ ratio $(\sim 0.040)$, in spite of its formation at high temperature, because its mother molecule, $\mathrm{CH}_{3} \mathrm{OH}$, is highly deuterated during the cold phase. It is also interesting that $\mathrm{CH}_{3} \mathrm{OCH}_{3}$ is under-abundant in our core model compared with observation, while its higher abundance in the pseudo-time dependent 
model is in much better agreement. The pseudo-time-dependent models also give higher

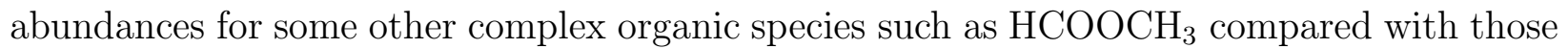
in the $1 \mathrm{D}$ core model. Since the size of the hot corino region $(T \geq 100 \mathrm{~K})$ is close to a typical centrifugal radius $(\sim 100 \mathrm{AU})$ of cloud cores, at least a fraction of the large organic species might be formed in such prolonged (i.e. longer than free-fall time scale) warm chemistry due to partial rotational support. Such predictions should be tested via observations at high spatial resolution.

We would like to thank the anonymous referee for useful comments to improve our manuscript. We are grateful to Prof. Naoki Watanabe for helpful discussions on grain-surface reactions. This work was supported by Grant-in-Aid for Scientific Research (A) 21244021, (C) 23540266, and Grant-in-Aid for Scientific Research on Innovative Areas 23103004. VW and FH thank the French CNRS/INSU program PCMI for its financial support. EH thanks the National Science Foundation (US) for his program in astrochemistry and NASA for the study of pre-planetary matter. Some kinetic data used here has been downloaded from the online database KIDA (KInetic Database for Astrochemistry, http://kida.obs.u-bordeaux1.fr).

\section{REFERENCES}

Adams, F.C. \& Shu, F.H. 1985, ApJ, 296, 655

Aikawa. Y., Ohashi, N., Inutsuka, S., Herbst, E. \& Takakuwa, S. 2001, ApJ 552, 639

Aikawa, Y., Wakelam, V., Garrod, R.T. \& Herbst, E. 2008, ApJ 674, 993

Alexander, D. R., \& Ferguson, J. W. 1994, ApJ, 437, 879

Alonso-Albi, T., Fuente, A., Crimier, N., Caselli, P., Ceccarelli, C., Johnstone, D., Planesas, P., Rizzo, J. R., Wyrowski, F., Tafalla, M., Lefloch, B., Maret, S. \& Dominik, C. 2010, A\&A, 518, 52

Andersson,S. \& van Dishoeck, E. F. A\&A, 491, 907

Arasa, C., Andersson, S., Cuppen, H. M., van Dishoeck, E. F. \& Kroes, G. J. 2011, JChPh, 134,164503

Atahan, A. Alexander, M.H. \& Rackham, J. 2005, J. Chem. Phys., 123, 204306

Bottinelli, S., Ceccarelli, C., Neri, R., Williams, J.P., Caux, E., Cazaux, S., Lefloch, B., Maret, S., Tielens, A.G.G.M. 2004, ApJ, 617, L69 
Caselli, P., Stantcheva, T., Shalabia, O., Shematovich, V.I. \& Herbst, E. 2002, P\&SS, 50, 1257

Cazaux, E., Tielens, A.G.G.M., Ceccarelli, C., Castets, A., Wakelam, V., Caux, E., Parise, B. \& Teyssier, D., 2003, ApJ, 593, L51

Cazaus, S., Caselli, P. \& Sppans, M. 2011, ApJL, 741, 34

Ceccarelli, C., Caselli, P., Herbst, E., Tielens, A. G. G. M., \& Caux, E. 2007, in Protostars and Planets V, ed. B. Reipurth, D. Jewitt, \& K. Keil (Tucson: Univ. Arizona Press), 47

Chandler, C.J., Brogen, C.L., Shirley, Y.L. \& Loinard, L. 2005, ApJ, 632, 371

Charnley, S., Tielens, A.G.G.M. \& Millar, T.J. 1992, ApJL 399, 71

Coutens, A., Vastel, C., Caux, E., Ceccarelli, C., Bottinelli, S., Wiesenfeld, L., Faure, A., Scribano, Y. \& Kahane, C. 2012, A\&A 539, 132

Fuente, A., Caselli, P., McCoey, C., Cernicharo, J., Johnstone, D., Fich, M., van Kempen, T., van Dishoeck, E., Yildiz, U., Visser, R., Kristensen, L., Alonso-Albi, T., Herpin, F. \& Tisi, S. 2012, A\&A, 540, 75

Furuya, K., Aikawa, Y., Sakai, N. \& Yamamoto, S. 2011, ApJ, 731, 38

Garrod, R.T. \& Herbst, E. 2006, A\&A 457, 927

Garrod, R. T., Wakelam, V., \& Herbst, E. 2007, A\&A, 467, 1103

Goumans, T.P.M. (2011), MNRAS 413, 2615

Harada, N., Herbst, E. \& Wakelam, V. 2010, ApJ, 721, 1570

Hasegawa, T. I., \& Herbst, E. 1993, MNRAS, 261, 83

Hassel, G.E., Herbst, E. \& Garrod R.T. 2008, ApJ, 681, 1385

Hassel, G.E., Harada, N., \& Herbst, E. 2011, ApJ, 743:182

Herbst, E. 1993, in Dust and Chemistry in Astronomy, T.J.Millar \& D.A. Williams eds, (Bristol: IOP), 183

Hersant, F., Wakelam, V., Dutrey, A., Guilloteau, S. \& Herbst, E. 2009, A\&A, 493, 49 
Hidaka, H., Watanabe, M., Kouchi, A. \& Watanabe, N. 2009, ApJ, 702, 291

Iglesias, C. A., \& Rogers, F. J. 1996, ApJ, 464, 943

Jørgensen, J.K. 2004, A\&A, 424, 589

Jørgensen, J.K. \& van Dishoeck 2010, ApJL, 725, 172

Keto, E. \& Caselli, P. 2010, MNRAS, 402, 1625

Kuan, Y.-J., Juang, H.-C., Charnley, S.B., Hirano, N., Takakuwa, S., Wilner, D.J., Liu, S.-Y., Ohashi, N., Bourke, T.L., Qi, C., \& Zhang, Q. 2004, ApJ, 616, L27

Lee, J.-E., Bergin, E.A. \& Evans, N.J.II 2004, ApJ 617, 360

Le Petit, F. \& Roueff, E. 2003, in Dissociative Recombination of Molecular Ions with Electrons, ed. Steven L. Guberman, (Dordrecht:Kluwer), 373

Liu, F.-C., Parise, B., Kristensen, L., Visser, R., van Dishoeck, E. F. \& Gusten, R. 2011, A\&A 527, 19

Maret, S. et al. 2004 A\&A 416577

Maret, S., Ceccarelli, C., Tielens, A.G.G.M., Caux, E., Lefloch, B., Faure, A., Castet, A., \& Flower, D.R. 2005 A\&A 442527

Margitan, J.J., Kaufman, F. \& Anderson, J.G. 1975, Chem, Phys. Letters, 34, 485

Masunaga, H., Miyama, S.M. \& Inutsuka, S. 1998, ApJ, 495, 346

Masunaga, H. \& Inutsuka, S. 2000, ApJ, 531, 350 ApJ

Millar, T.J., Bennet, A. \& Herbst E. 1989, ApJ, 340, 906

Munoz Caro, G. M., Jimenez-Escobar, A., Martin-Gago, J. A., Rogero, C., Atienza, C., Puertas, S., Sobrado, J. M. \& Torres-Redondo, J. 2010, A\&A, 522, 108

Nagaoka, A., Watanabe, N., Kouchi, A. 2007, J. Phys. Chem. A 2007, 111, 3016

Nagaoka, A., Watanabe, N., Kouchi, A. 2005, ApJ 624, L29

Nomura, H., Aikawa, Y., Nakagawa, Y. \& Millar, T. J. 2009, A\&A 495, 183

Öberg, K.I., van Dishoeck, E.F. \& Linnartz, H. 2009, A\&A, 496, 281

Öberg, K.I., Linnartz, H., R. Visser \& van Dishoeck, E.F. 2009, A\&A, 693, 1209 
Öberg, K.I., van der Marel, N., Kristensen, L.E., \& van Dishoeck, E.F. 2011, ApJ, 740, 14

Parise, B., Simon, T., Dartise, E., Ceccarelli, C., Rayner, J. \& Tielens, A.G.G.M. 2003, A\&A, 410, 897

Parise, B., Castets, A., Herbst, E., Caux, E., Ceccarelli, C., Mukhopadhyay, I., Tielens, A.G.G.M. 2004, A\& A, 416, 159

Parise, B., Caux, E., Castets, A., Ceccarelli, C., Loinard, L., Tielens, A. G. G. M., Bacmann, A., Cazaux, S., Comito, C., Helmich, F., Kahane, C., Schilke, P., van Dishoeck, E., Wakelam, V., Walters, A. 2005, A\&A 431, 547

Parise, B., Ceccarelli, C., Tielens, A.G.G.M., Catet, A., Caux, E., Lefloch, B. \& Maret, S. 2006, A\&A 453, 949

Pineda, J., Maury, A.J., Fuller, G.A., Testi, L., Garcia-Appadoo, D., Peck, A. B., Villard, E., Corder, S. A., van Kempen, T.A., Turner, J. L., Tachibana, K., Dent, W. 2012, A\& A 544, L7

Piskunov, N., Wood, B. E., Linsky, J. L., Dempsey, R. C., \& Ayres, T. R. 1997, ApJ, 474, 315

Preibisch, Th., Ossenkopf, V., Yorke, H. W., \& Henning, Th. 1993, A\&A, 279, 577

Remijian, A.J. \& Hollis, J.H. 2006, ApJ, 640, 842

Roberts, H., Herbst, E. \& Millar, T.J. 2004, A\&A, 424, 905

Rodgers, S.D. \& Charnley, S.B. 2003, ApJ, 585, 355

Rouef, E., Lis, D.C., can der Tak, F.F.S., Gerin, M., \& Goldsmith, P.F. 2005, A\&A 438, 585

Ruffle, D.P. \& Herbst, E. 2000, MNRAS, 319, 837

Sakai, N., Sakai, T., Hirota, T., \& Yamamoto, S. 2008, ApJ, 672, 371

Sakai, N., Sakai, T., Hirota, T. \& Yamamoto, S. 2009, ApJ, 702, 1025

Sakai, N., Sakai, T., Hirota, T. \& Yamamoto, S. 2010, ApJ, 722, 1633

Schöier, F. L., Jørgensen, J. K., van Dishoeck, E. F. \& Blake, G. A. 2002, A\&A 390, 1001

Semenov, D., Hersant, F., Wakelam, V., Dutrey, A., Chapillon, E., Guilloteau, St., Henning, Th., Launhardt, R., Pietu, V. \& Schreyer, K. 2010, A\&A, 522, 42 
Shu, F. 1977, ApJ, 214, 488

Spitzer, L.Jr. 1978, Physical Processes in the Interstellar Medium (New York: John Wiley \& Sons)

Stantcheva, T., Caselli, P., \& Herbst, E. 2001, A\&A, 375, 673

Taquet, V., Caccarelli, C. \& Kahane, C. 2012, ApJL, 748, L3

van Dishoeck, E.F. \& Black, J.H. 1988, ApJ, 334, 771

Visser, R., Doty, S.D., \& van Dishoeck, E.F. 2011, A\&A 534, 132

Watanabe, N. \& Kouchi, A. 2008, Progress in Surface Science, 83, 439

Watanabe, N., Kimura, Y., Kouchi, A., Chigai, T., Hama, T. \& V. Pirronello, ApJL, 714, 233

Williams, D. A. 1968, ApJ, 151, 935

Yildiz, U.A., Kristensen, L. E., van Dishoeck, E.F., Belloche, A., van Kempen, T.A., Hogerheijde, M.R., Gusten, R., van der Marel, N. 2012, A\&A, 542, 86 

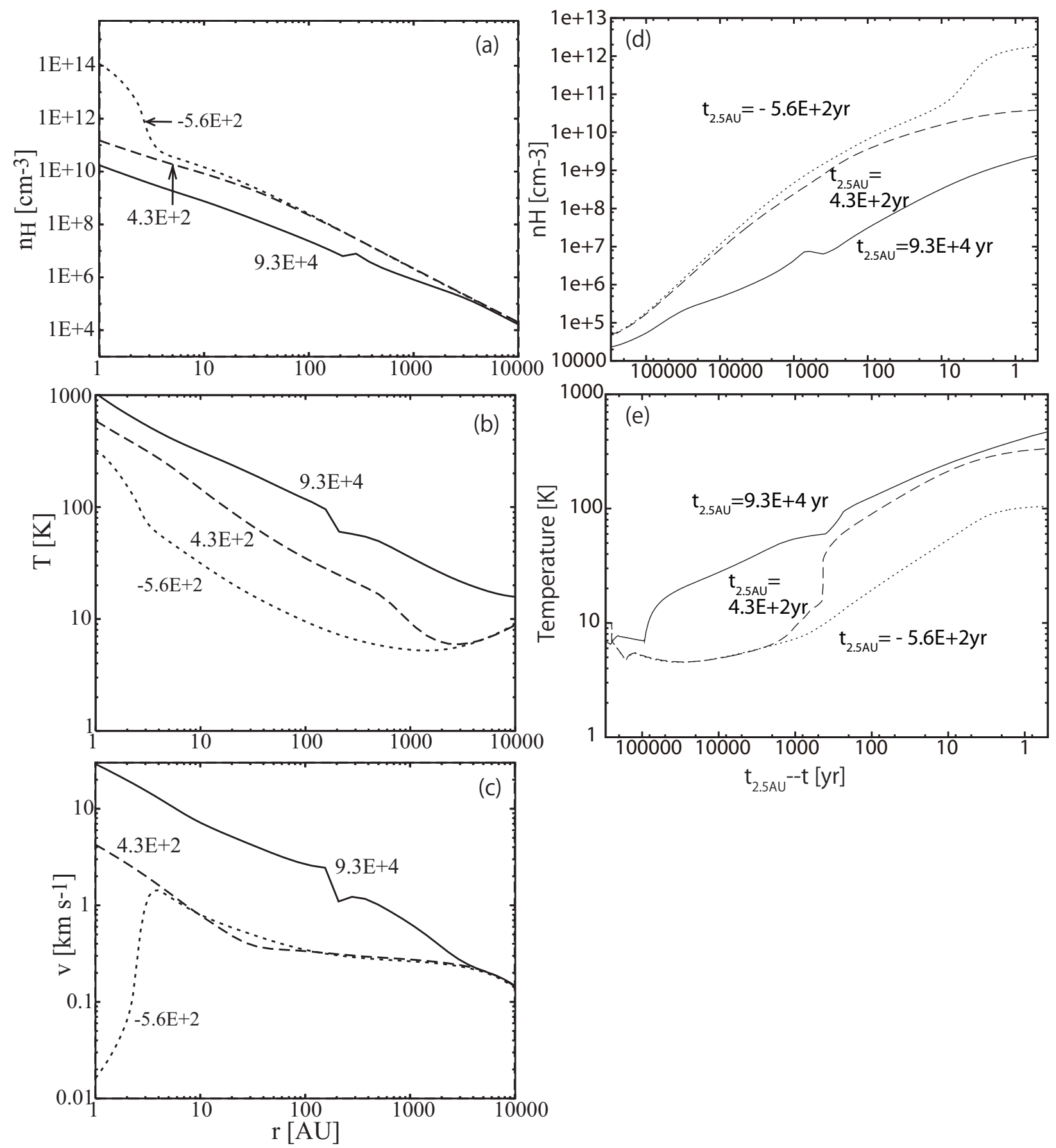

Fig. 1.- $(a-c)$ Radial distribution of density $\left(n_{\mathrm{H}}\right)$, temperature and infalling velocity at $t=-5.6 \times 10^{2} \mathrm{yr}$ (dotted line), $4.3 \times 10^{2} \mathrm{yr}$ (dashed line), and $9.3 \times 10^{4} \mathrm{yr}$ (solid line), where $t=0$ is defined by the birth of a protostar. $(d-e)$ Temporal variation of density and temperature in fluid parcels which reach $r=2.5 \mathrm{AU}$ at $t=-5.6 \times 10^{2} \mathrm{yr}, 4.3 \times 10^{2} \mathrm{yr}$, and $9.3 \times 10^{4} \mathrm{yr}$. 


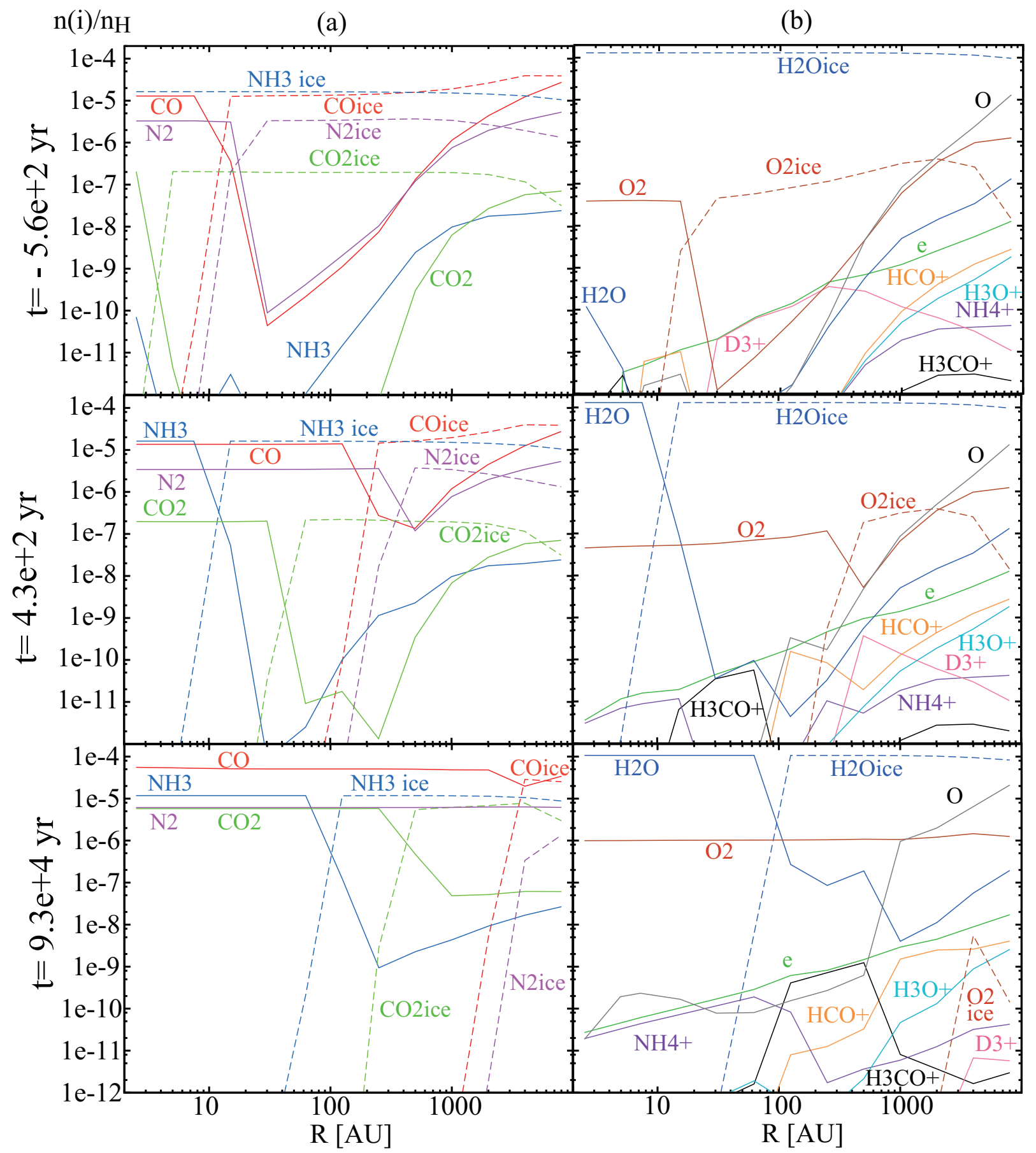

Fig. 2.- Radial distribution of simple molecules $(a-b)$, complex organic species $(c)$, and carbon chain species $(d)$ at $t=-5.6 \times 10^{2} \mathrm{yr}, 4.3 \times 10^{2} \mathrm{yr}$, and $9.3 \times 10^{4} \mathrm{yr}$. Solid lines and dashed lines depict gas-phase species and ice mantle species, respectively, while the chemical species are distinguished by line colors. Note that the horizontal axis starts from 2 AU rather than 1 AU. 


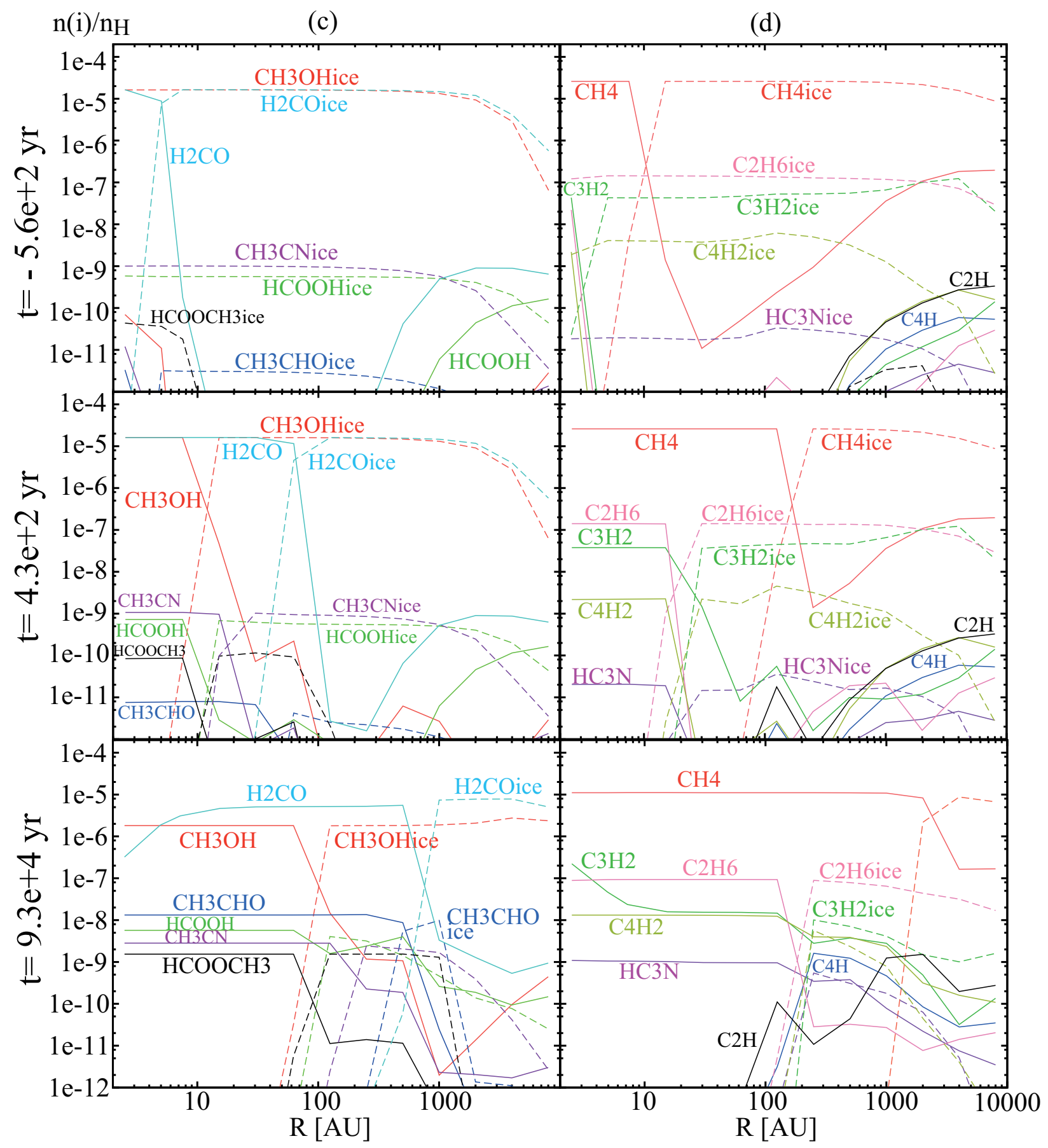

Fig. 2.- (cont.) 

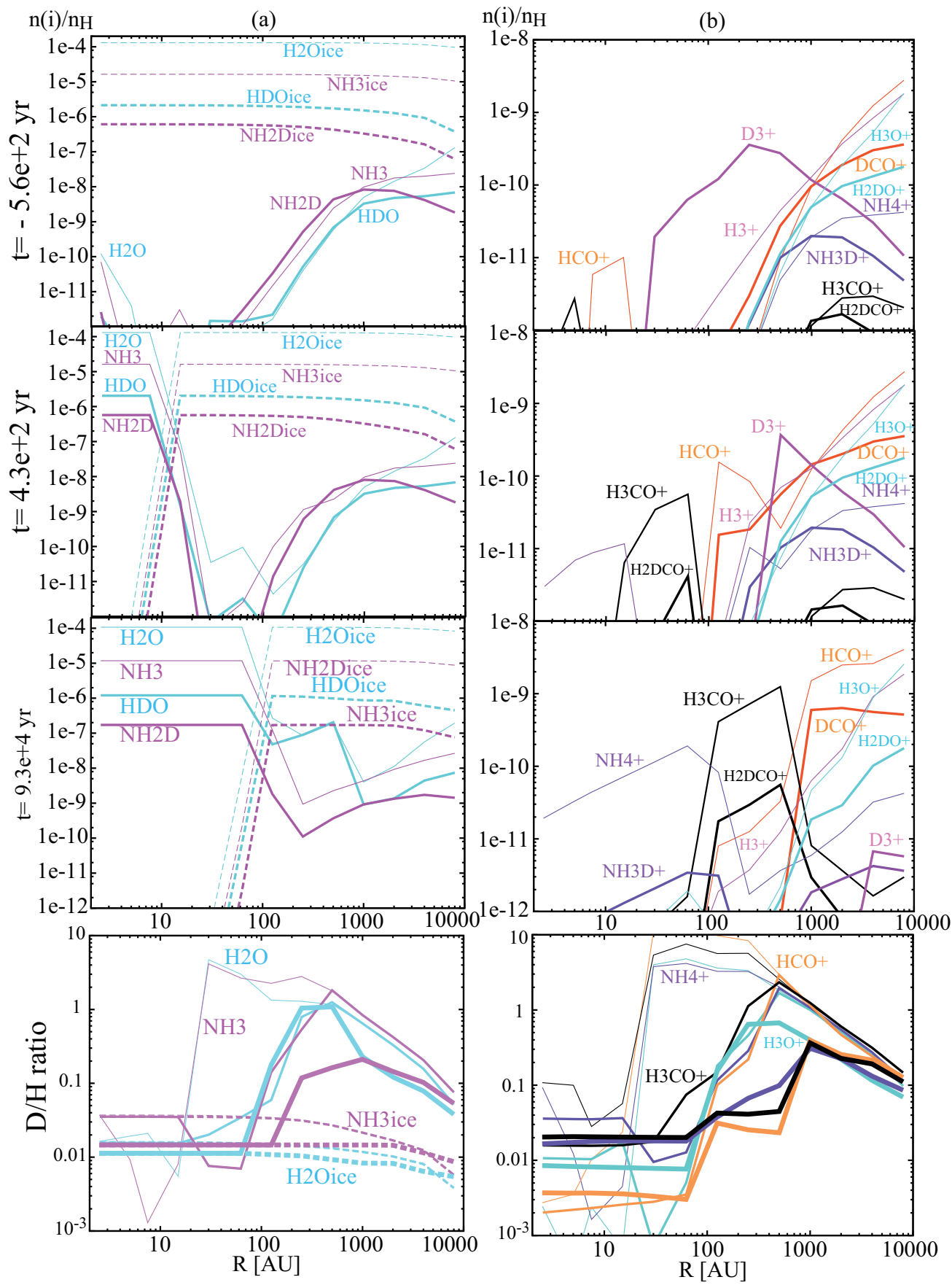

Fig. 3.- Radial distribution of mono-deuterated species and their normal isotope counterparts at $t=-5.6 \times 10^{2} \mathrm{yr}, 4.3 \times 10^{2} \mathrm{yr}$, and $9.3 \times 10^{4} \mathrm{yr}$. The bottom panels show the radial distribution of the $\mathrm{D} / \mathrm{H}$ ratio for assorted molecules. The thin, thick, and bold lines depict the $\mathrm{D} / \mathrm{H}$ ratios at $t=-5.6 \times 10^{2} \mathrm{yr}, 4.3 \times 10^{2} \mathrm{yr}$, and $9.3 \times 10^{4} \mathrm{yr}$, respectively. 


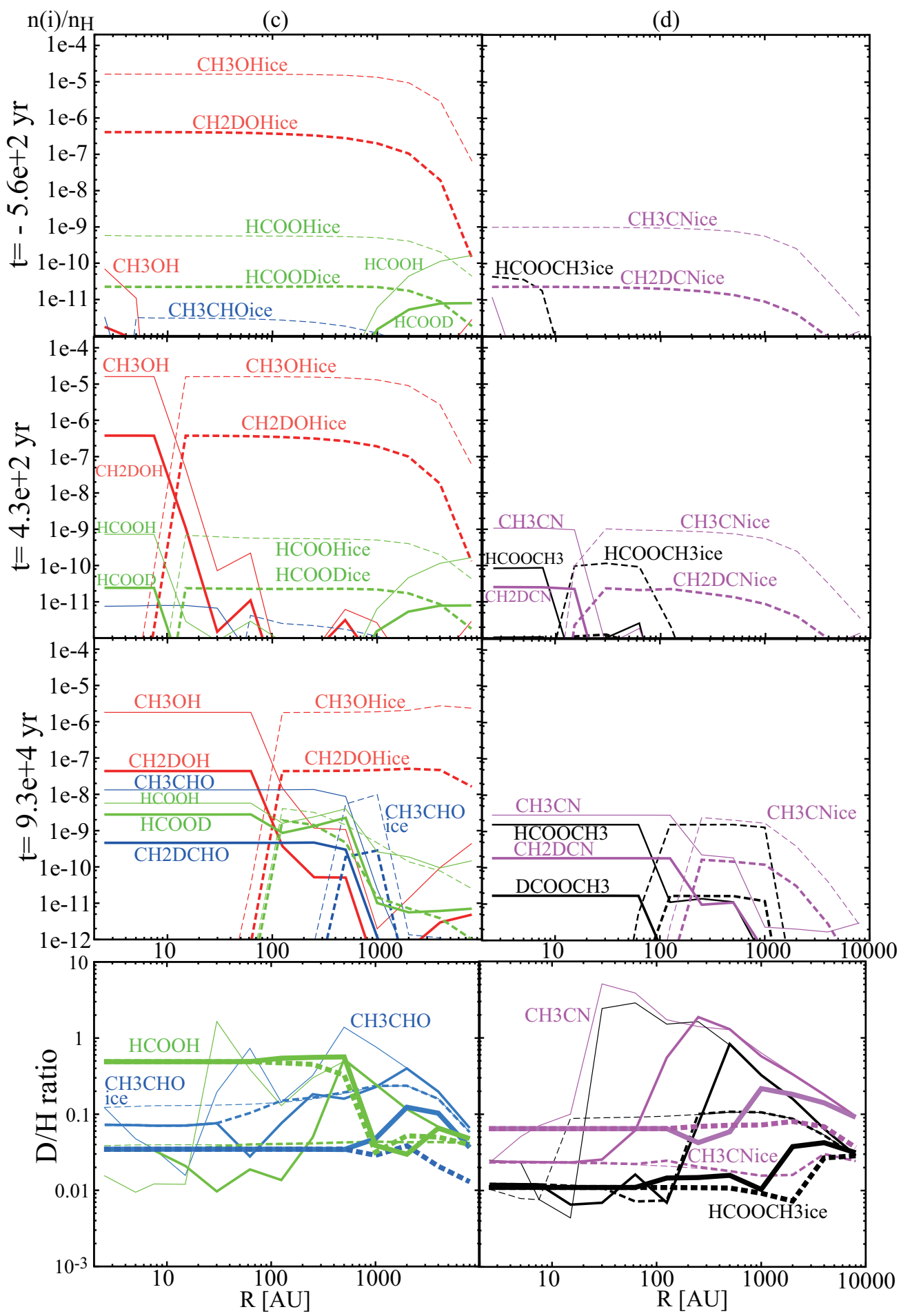

Fig. 3.- cont. 

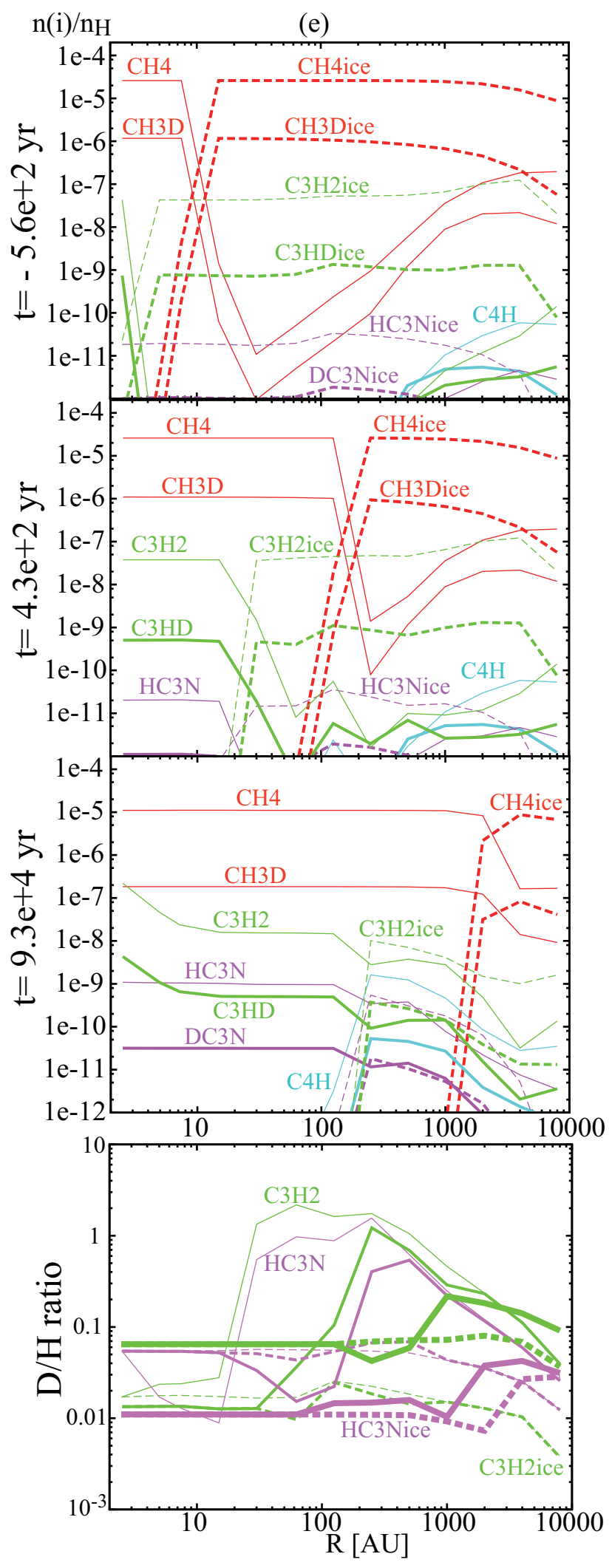

Fig. 3.- cont. 


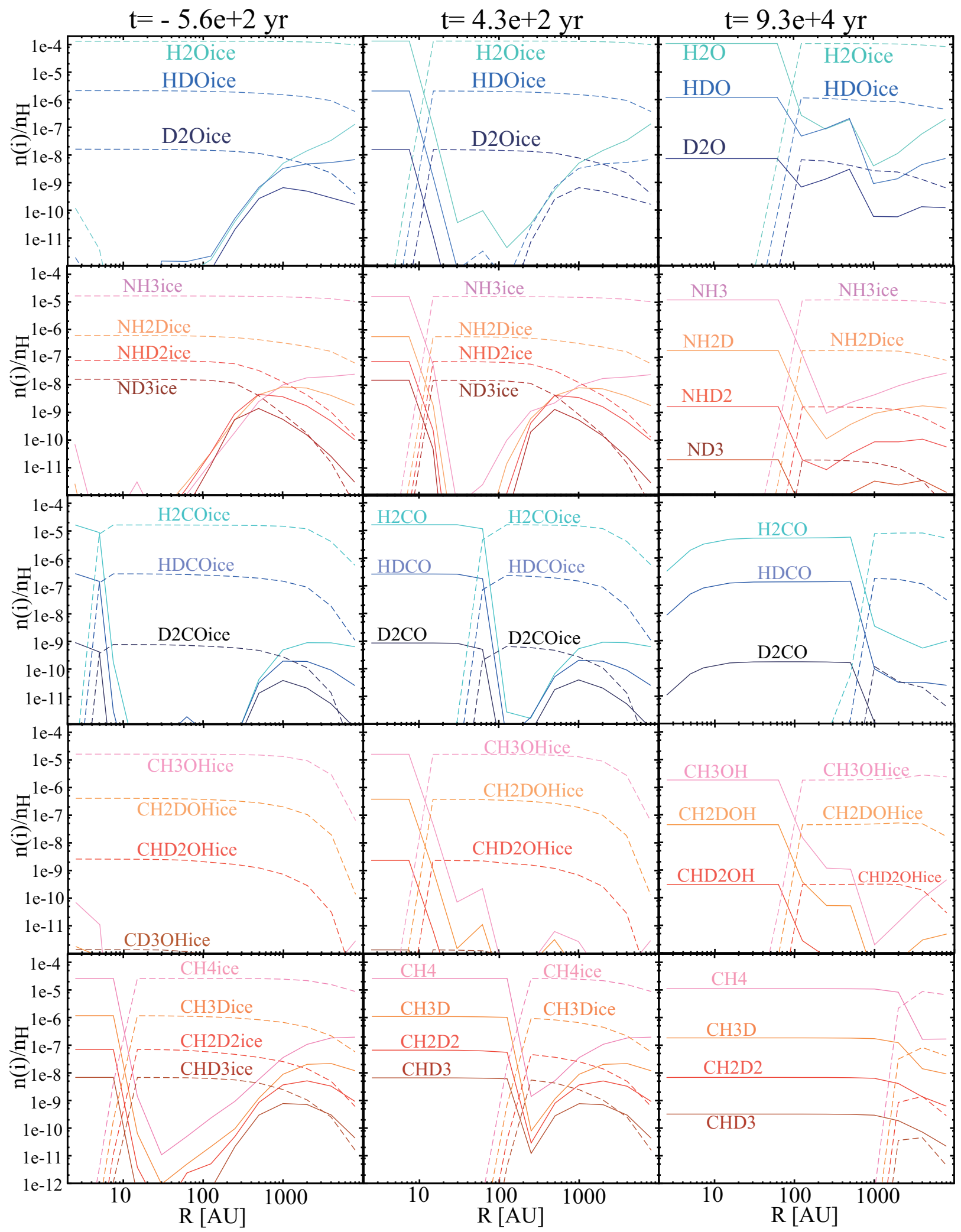

Fig. 4.- Radial distribution of mono-, multi-deuterated species and their normal isotope counterparts at $t=-5.6 \times 10^{2} \mathrm{yr}, 4.3 \times 10^{2} \mathrm{yr}$, and $9.3 \times 10^{4} \mathrm{yr}$. 


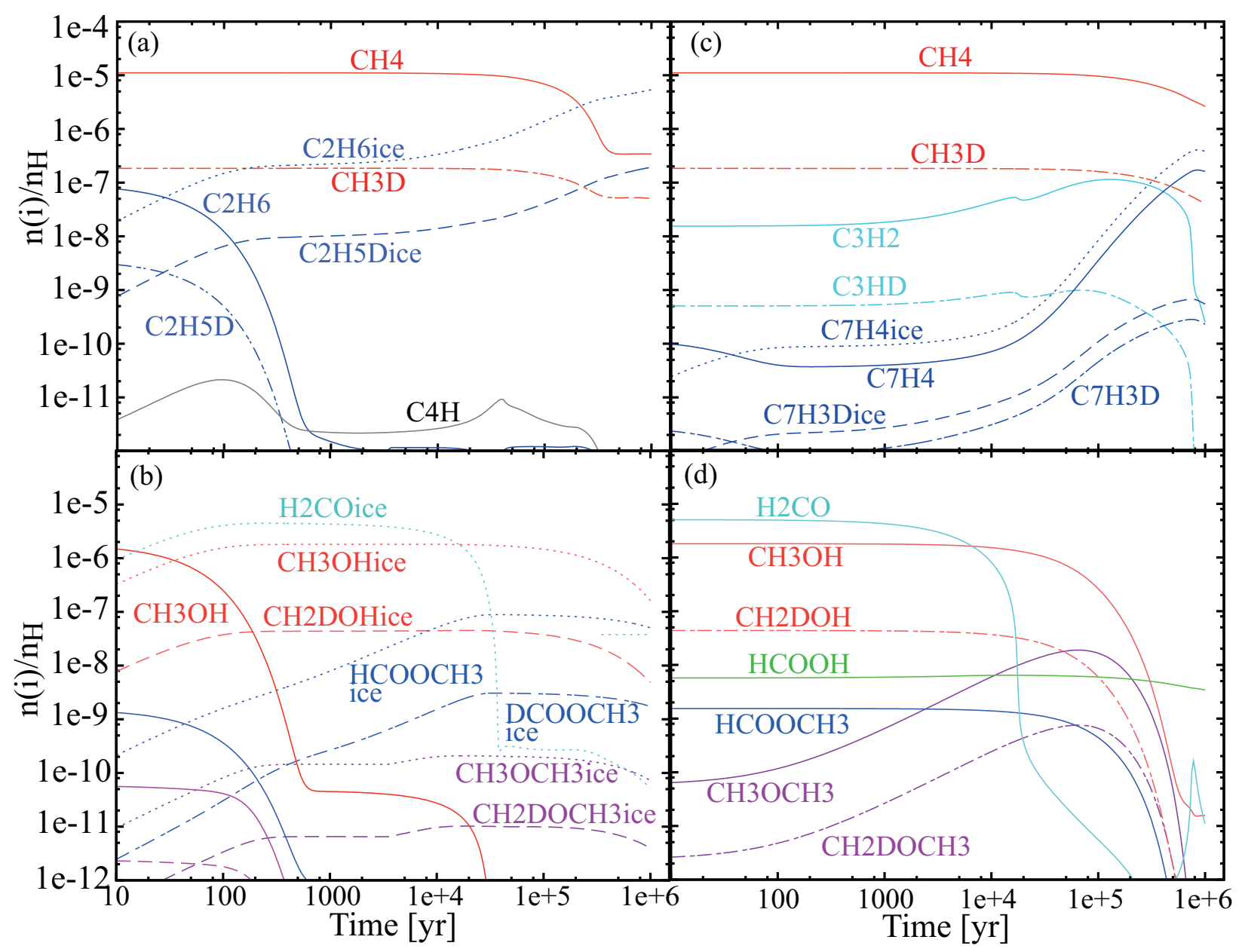

Fig. 5.- Pseudo-time-dependent models with $n_{\mathrm{H}}=1.487 \times 10^{8} \mathrm{~cm}^{-3}, T=40 \mathrm{~K}$ (panels a, b) and $T=150 \mathrm{~K}$ (panels c, d). The initial abundance is set by the $1 \mathrm{D}$ model at $t=9.3 \times 10^{4}$ yr and $r=30$ AU. 


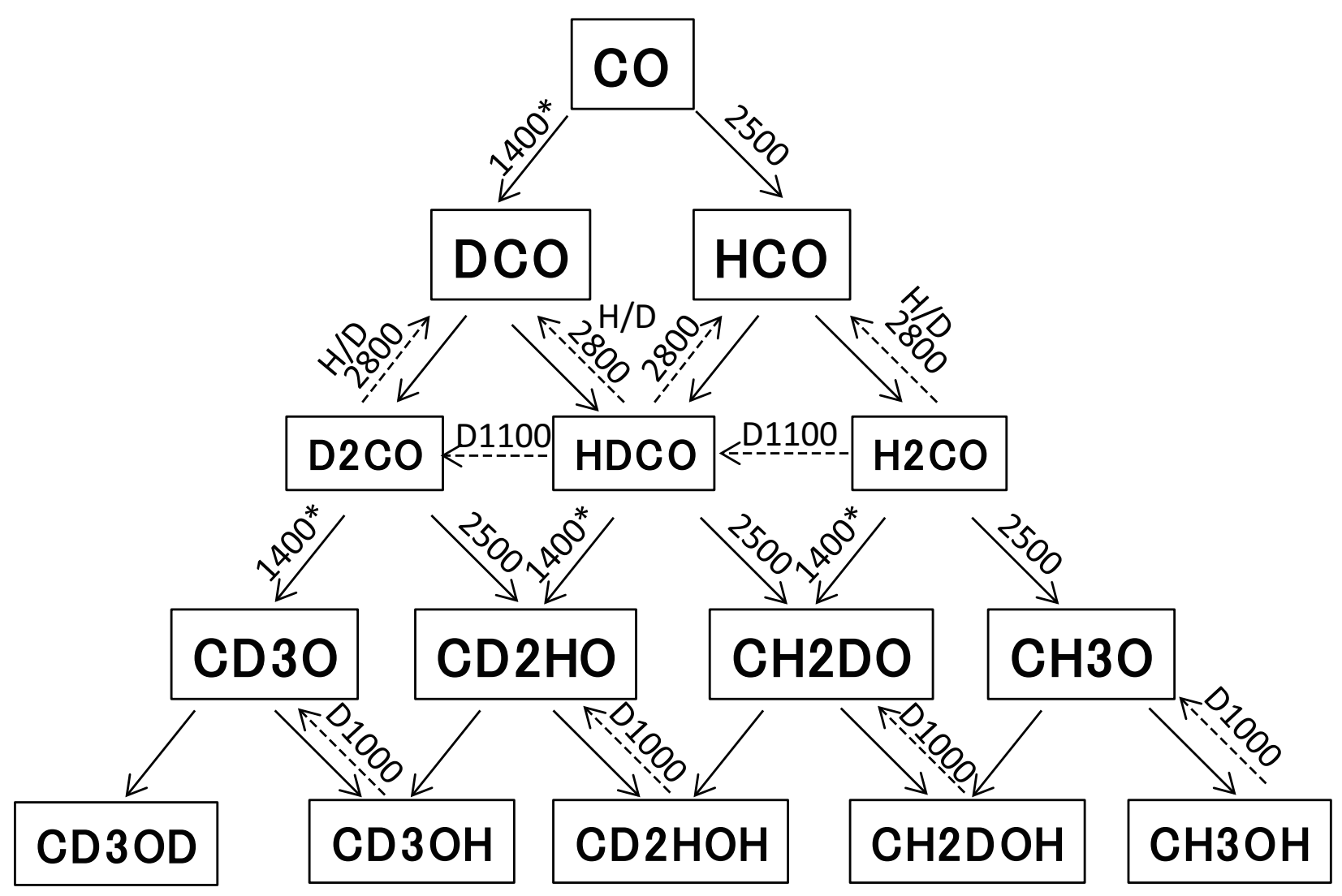

Fig. 6.- Reaction network of $\mathrm{H}_{2} \mathrm{CO}$ and $\mathrm{CH}_{3} \mathrm{OH}$ formation on the grain surface. Solid arrows refer to the reactions in our original model, while the dashed arrows are newly added reactions. The $\mathrm{H}$ or $\mathrm{D}$ and values represent the reactants and activation barrier heights expediently assumed for the tunneling of our rectangular potential. For D atom reactions with asterisks, we lowered the activation barrier so that their rates are about $10 \%$ of the $\mathrm{H}$ atom reactions. 


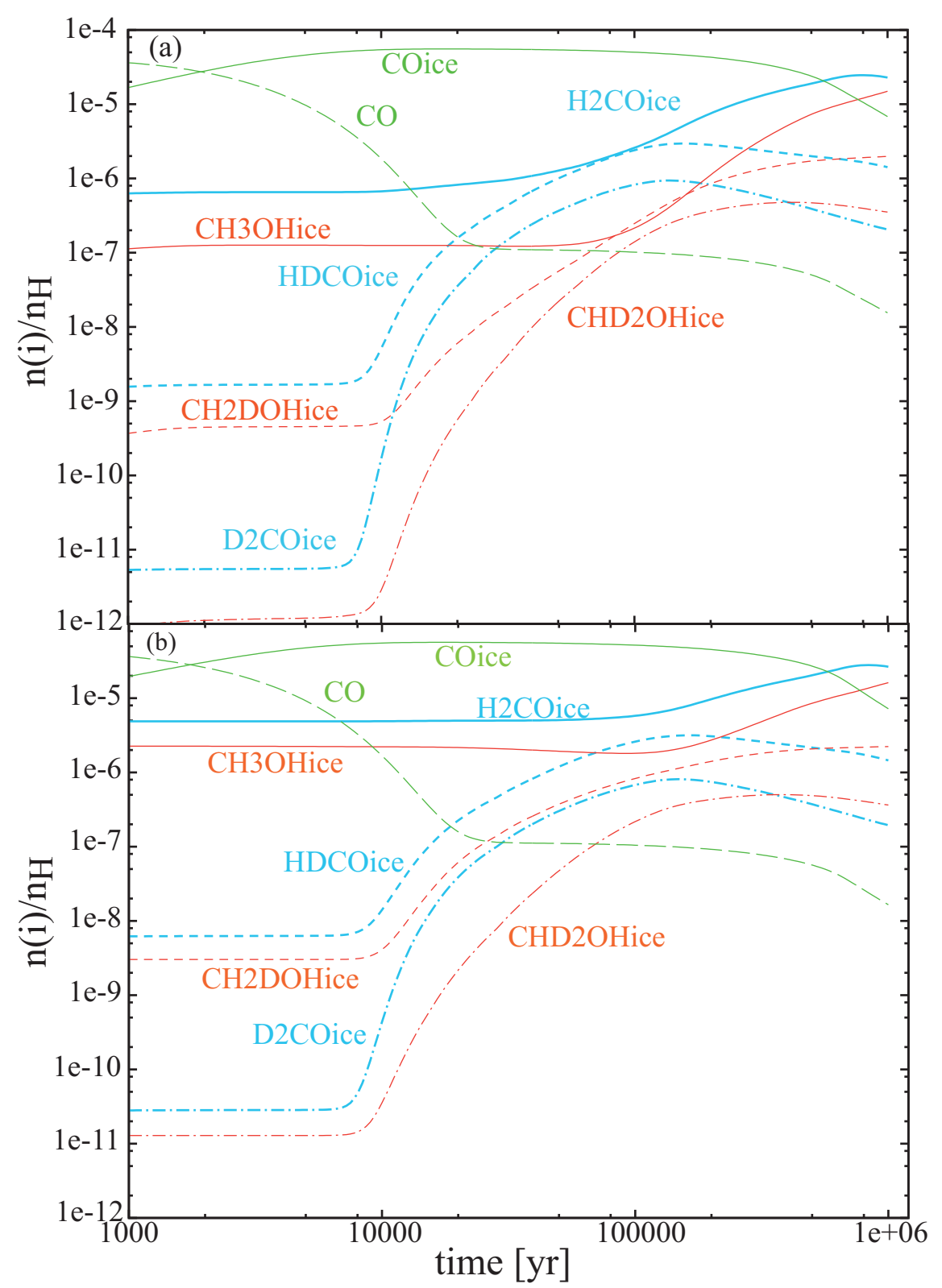

Fig. 7.- Pseudo-time-dependent model at $n_{\mathrm{H}}=5 \times 10^{6} \mathrm{~cm}^{-3}$ and $T=10 \mathrm{~K}$ with the substitution and abstraction reactions. The initial abundance is determined by calculating the molecular evolution at molecular cloud conditions for $8 \times 10^{4} \mathrm{yr}(\mathrm{a})$ and $2 \times 10^{5} \mathrm{yr}(\mathrm{b})$. 